\title{
The latent words language model ${ }^{\text {is }}$
}

\author{
Koen Deschacht, Jan De Belder, Marie-Francine Moens* \\ K.U.Leuven, Department of Computer Science, Celestijnenlaan 200A, B-3001 Heverlee, Belgium
}

Received 26 March 2012; accepted 5 April 2012

Available online 20 April 2012

\begin{abstract}
We present a new generative model of natural language, the latent words language model. This model uses a latent variable for every word in a text that represents synonyms or related words in the given context. We develop novel methods to train this model and to find the expected value of these latent variables for a given unseen text. The learned word similarities help to reduce the sparseness problems of traditional n-gram language models. We show that the model significantly outperforms interpolated Kneser-Ney smoothing and class-based language models on three different corpora. Furthermore the latent variables are useful features for information extraction. We show that both for semantic role labeling and word sense disambiguation, the performance of a supervised classifier increases when incorporating these variables as extra features. This improvement is especially large when using only a small annotated corpus for training.
\end{abstract}

(c) 2012 Elsevier Ltd. All rights reserved.

Keywords: Language model; Information extraction; Word sense disambiguation; Semantic role labeling

\section{Introduction}

Since the very beginning of the field of natural language processing, researchers have tried to create models of natural language that offer a computer program a mean of generating, manipulating and classifying a given text. Early models were constructed by hand and were limited to a small domain (Winograd, 1972). It was soon found that these models were too rigid for natural language that contains a lot of variation that is not easily captured in fixed rules (Winston, 1976). With the introduction of large, hand-annotated corpora (e.g. the Penn treebank (Marcus et al., 1994)), most researchers turned to shallow methods for the classification of natural language. Examples of these include support vector machines (Cortes and Vapnik, 1995), hidden Markov models (Baum et al., 1970), maximum entropy classifiers (Berger et al., 1996) and others, which have been successfully used for many tasks, including named entity recognition (Sang and De Meulder, 2003), part-of-speech labeling (Brants, 2000) and phrase chunking (Kudo and Matsumoto, 2001). Limitations of this approach are however that for every new task a new corpus needs to be manually annotated, which is very costly both in man-hours, and the required crafting of features by hand that capture correlations between the input data and the output data.

\footnotetext{
is This paper has been recommended for acceptance by 'Edward J. Briscoe'.

* Principal corresponding author.

E-mail addresses: koendeschacht@gmail.com (K. Deschacht), Jan.DeBelder@cs.kuleuven.be (J. De Belder), Marie-Francine.Moens@cs.kuleuven.be (M.-F. Moens).
} 
Table 1

Output of the LWLM on an example sentence, showing the most probable hidden words (bottom rows, sorted according to descending probability) for a given observed sentence (top row) from the Reuters training corpus.

\begin{tabular}{|c|c|c|c|c|c|c|c|}
\hline $\mathrm{a}$ & japanese & electronics & executive & was & kidnapped & in & mexico \\
\hline a & japanese & tobacco & executive & was & kidnapped & in & mexico \\
\hline the & u.s. & electronics & director & is & abducted & on & usa \\
\hline its & german & sales & manager & were & killed & at & uk \\
\hline a & british & consulting & economist & are & found & of & australia \\
\hline one & russian & electric & spokesman & be & abduction & into & canada \\
\hline
\end{tabular}

Ideally we would like to develop models that have some deeper understanding of natural language. Large improvements in computational speed and storage size, together with the availability of enormous unlabeled corpora, have sparked an interest in unsupervised models that learn structures or statistics from unlabeled texts. Examples of these are topic models (Blei et al., 2003; Griffiths et al., 2005), unsupervised part-of-speech taggers (Goldwater and Griffiths, 2007) and deep neural networks (Collobert and Weston, 2008). Research has shown that the statistics or structures learned by these models can be successfully used in many supervised tasks, such as document classification (Blei et al., 2003), part-of-speech tagging, word segmentation in Chinese ( $\mathrm{Li}$ and McCallum, 2005), dependency parsing (Koo et al., 2008) and named entity recognition (Miller et al., 2004). However, till now no unsupervised, generative model was proposed that learns accurate, fine-grained semantic similarities between words.

In this article we develop the latent words language model (LWLM). This model is a novel statistical model that learns context dependent hidden (or latent) words from unlabeled texts. A hidden word of a certain word in a specific textual context is a variable in a Bayesian network that generates the word. This variable models the probability distribution of every word in the vocabulary of the corpus on which the model is trained, indicating the probability that the concerned vocabulary word occurs in the considered context. Words that have a high probability of replacing the original word in the given context are often synonyms or have at least a strong semantic relationship with the original word. Table 1 shows the actual output of the model on an example sentence. This model has the following attractive properties: (1) it is trained on unlabeled texts, which are abundantly available in electronic format for most languages and do not require any manual annotation, (2) it does not require any knowledge of the syntactic or semantic structure of the text, and as such is completely language independent ${ }^{1}$ and (3) it learns fine-grained semantic classes (i.e. the hidden words) that capture semantic and syntactic similarities.

The learned hidden words can be used to overcome the sparseness problem fundamental to natural language processing. We show this in three applications. In a first application we incorporate the hidden words in a language model and show that it outperforms Kneser-Ney and cluster language models on three different corpora. We then use the hidden words into a semantic role labeling system and word sense disambiguation system. For both systems this extra knowledge improves the accuracy significantly. The resulting word sense disambiguation system outperforms all other published systems on the corpus of the Senseval3 workshop. Furthermore the hidden words are included using a simple method (i.e. as extra features), which generalizes to virtually all feature-based natural language processing systems.

We fully describe the LWLM in Section 2. The LWLM is heavily influenced by the existing work on n-gram language models, and we first introduce these models in Section 2.1. Then we develop methods for training and inference the LWLM, and compare it to existing language models. We will see in Section 3 that the latent variables learned in this model can be successfully employed in supervised information extraction tasks, which is demonstrated by a semantic role label and a word sense disambiguation classifier. Finally we discuss related work in Section 4 and conclude this article in Section 5.

\footnotetext{
${ }^{1}$ We assume that the words in the text have been tokenized. This is reasonable for most languages, although not fully solved for some, such as Japanese and Chinese
} 


\section{The latent words language model}

\subsection{Introduction to language models}

Language models are statistical models that assign a probability to every sequence of words $\mathbf{w}=\left[w_{1} \ldots w_{N_{t}}\right]$, ideally reflecting the probability that this sequence is used by a human user of language. These models have been used in a wide range of applications, such as speech recognition (Jelinek et al., 1975), machine translation (Brown et al., 1990), spelling correction (Kemighan et al., 1990) and handwriting recognition (Srihari and Baltus, 1992). The most successful class of language models are n-gram models, which were introduced almost three decades ago (Bahl et al., 1983). n-Gram language models estimate the probability $P(\mathbf{w})$ of the sequence of words $\mathbf{w}=\left[w_{1} \ldots w_{N_{t}}\right]$ of length $N_{t}$ as

$$
P(\mathbf{w})=\prod_{i=1}^{N_{t}} P\left(w_{i} \mid \mathbf{w}_{i-n+1}^{i-1}\right)
$$

where $\mathbf{w}_{i-n+1}^{i-1}=\left[w_{i-n+1} \ldots w_{i-1}\right]$ is the sequence of $n-1$ words that occur before $w_{i}$. The value of $n$ is usually set to a small number (e.g. 3). These models are trained on a large unlabeled corpus $\mathbf{w}_{\text {train }}=\left[w_{1} \ldots w_{N_{t}}\right]$. Let us first consider the maximum likelihood estimate of the probability $P\left(w_{i} \mid \mathbf{w}_{i-n+1}^{i-1}\right)$, given by

$$
P_{M L}\left(w_{i} \mid \mathbf{w}_{i-n+1}^{i-1}\right)=\frac{c\left(\mathbf{w}_{i-n+1}^{i}\right)}{c\left(\mathbf{w}_{i-n+1}^{i-1}\right)}
$$

where $c\left(\mathbf{w}_{i-n+1}^{i}\right)$ is the number of times the sequence of words $\mathbf{w}_{i-n+1}^{i}$ occurs in $\mathbf{w}_{\text {train }}$ and $c\left(\mathbf{w}_{i-n+1}^{i-1}\right)$ is the number of times the sequence $\mathbf{w}_{i-n+1}^{i-1}$ occurs in $\mathbf{w}_{\text {train }}$. This estimate results in an ill-defined model, since it assigns zero probability to many sequences. The reason for this is that an exponential number of $\mathrm{n}$-grams can occur in a given corpus (i.e. $|V|^{n}$ with $|V|$ the size of the vocabulary, usually between $10^{4}$ and $10^{6}$ words). An unobserved test corpus is thus likely to contain many sequences that have never been observed. Smoothing methods overcome this problem generally by combining the probability of $\mathbf{w}_{i-n}^{i}$ with lower order probabilities, i.e. the probability of observing $\mathbf{w}_{i-n+1}^{i}$, the probability of observing $\mathbf{w}_{i-n+2}^{i}, \ldots$ and the probability of observing $w_{i}$. Chen and Goodman (1996) have compared a large number of smoothing techniques and found that Kneser-Ney smoothing (Ney et al., 1994) consistently outperformed all other smoothing methods. We use Kneser-Ney smoothing in our work, and expand it to non-discrete counts such as found in the probabilistic models of Section 2.3.2.

An important limiting factor of the performance of n-gram language models is the sparse nature of $n$-grams. Although smoothing methods partially alleviate this problem, they do not fully solve it. A major weakness of these models is that they treat every word in the text as a unique symbol, independent of all other symbols. This ignores the fact that many words are synonyms or have related meanings, and that natural language typically uses constructions where certain classes of words (e.g. syntactic classes) typically occur in the same order (e.g. "determiner noun verb"). Let us assume for example that we observe the sequence "let's meet on Tuesday" in the training corpus. If the model would know that "Tuesday" is similar to "Monday", "Wednesday" etc., we could predict that the sequences "let's meet on Monday", "let's meet on Wednesday", etc. can also be observed in the test corpus.

In this section we build a model that aims at exactly this goal: to learn words that are synonyms or that have related meanings, and use these in an improved model for the prediction of sequences in the test corpus. We first describe the structure of the model in Section 2.2. In this model we employ Kneser-Ney smoothing, which we describe in Section 2.3 together with a novel and improved variant that can be used with probabilistic counts. In Section 2.4.1 we will see that the standard method for inference is unfeasible due to a large number of values for the hidden variables, and we develop two approximate inference methods: the forward-forward beam search (Section 2.4.2), and a Gibbs sampling method (Section 2.4.3). These algorithms are then used to train the model on a large unlabeled corpus (Section 2.5). In Section 2.6 we discuss how to compute the probability of an unseen text. Finally we compare the LWLM with several state-of-the-art language models in Section 2.7. 


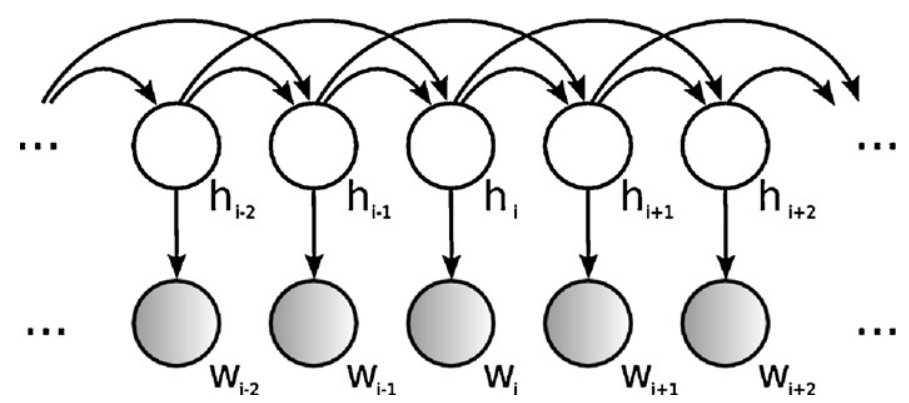

Fig. 1. Bayesian network representation of the latent words language model. The words $w_{i}$ (gray nodes) are observed and the hidden words $h_{i}$ (white nodes) are hidden variables, arrows indicate directed dependencies.

\subsection{Description of the latent words language model}

The latent words language model (LWLM) introduces, for a text $\mathbf{w}=\left[w_{1} \ldots w_{N_{t}}\right]$ of length $N_{t}$, a hidden (or latent) word $h_{i}$ for every observed word $w_{i}$ at position $i$. The value of $h_{i}$ is unknown but drawn from the vocabulary $V$. This model is a generative model for natural language that, for a given vocabulary $V$, length $N$, counts $C$ and smoothing parameters $\gamma$, generates a sequence of hidden symbols $\mathbf{h}=\left[h_{1} \ldots h_{N_{t}}\right]$ and a sequence of observed words $\mathbf{w}=\left[w_{1} \ldots w_{N_{t}}\right]$. The generative process is defined as follows:

For $i$ from 1 to $N_{t}$ do

Sample a hidden word $h_{i}$ from the distribution $P\left(h_{i} \mid \mathbf{h}_{i-n+1}^{i-1}, C, \gamma\right)$

Sample an observed word $w_{i}$ from the distribution $P\left(w_{i} \mid h_{i}, C, \gamma\right)$

Here we implicitly understand that for the first words in the sequence, i.e. for $i<n$, we only use the available context, e.g. if $i=1$ then $P\left(h_{i} \mid \mathbf{h}_{i-n+1}^{i-1}, C, \gamma\right)=P\left(h_{i} \mid C, \gamma\right)$. This model uses two probability distributions: the first distribution, $P\left(h_{i} \mid \mathbf{h}_{i-n+1}^{i-1}, C, \gamma\right)$, models the dependency between the current hidden word and the previous hidden words, and the second distribution, $P\left(w_{i} \mid h_{i}, C, \gamma\right)$ models the dependency between the observed word and the hidden word. Both probability distributions are categorical ${ }^{2}$ distributions smoothed with a variant of Kneser-Ney smoothing (see Section 2.3).

Given a sequence of observed words $\mathbf{w}=\left[w_{1} \ldots w_{N_{t}}\right]$ and hidden words $\mathbf{h}=\left[h_{1} \ldots h_{N_{t}}\right]$, the collection $C$ contains the collection of counts collected from these sequences, more specifically it contains for every hidden word $h_{i}$ the frequency of the word $w_{i}$ generated by that hidden word, and it contains for every sequence of hidden words $h_{i-m+1} \ldots h_{i}$, with length $m$ ranging from $n$ to 1 , the frequency of this sequence in $\mathbf{h}=\left[h_{1} \ldots h_{N_{t}}\right]$. The collection $\gamma$ contains the collection of discount factors used in these two distributions, which are often referred to in this article as the "smoothing parameters".

One interpretation of this model states that a person who wishes to express a certain message, can choose a large number of ways of expressing this message. However, when the message is uttered (or written down), this person has to chose one specific sequence of words, although many words in this sequence could be replaced with a synonym or related word that would keep the meaning of the message intact. The hidden word $h_{i}$ represents these possible alternative words at a certain position $i$ in this sequence. It is modeled as a probability distribution $P\left(h_{i} \mid \mathbf{w}\right)$ over all words in the vocabulary. We will see that by training this model on a large collection of unlabeled texts, we can learn word similarities with a high accuracy. These word similarities can then be used successfully in models of natural language and in information extraction methods, such as word sense disambiguation and semantic role labeling.

The structure of the Bayesian network expressed by the LWLM is shown in Fig. 1. From this figure we see that the structure of the model is equivalent to the structure of a hidden Markov model (HMM, see Baum et al., 1970; Baker, 1975). However, the two models have important differences: where the latent variables in a HMM are selected from a small set of categories, we model the latent variables as unseen words that can be selected from the entire vocabulary.

\footnotetext{
${ }^{2}$ Other authors use the term "multinomial distribution" to refer to the categorical distribution (e.g. Blei et al., 2003).
} 
Furthermore we use a novel smoothing method (i.e. RDKN, see Section 2.3.2) to compute the probability distributions, and where HMM's typically assume that the hidden variables are only dependent on the previous variable (i.e. $n=2$ ), we use much longer dependencies (i.e. $n \geq 5$ ), which has important consequences for the algorithms employed for training and inference. We will further compare the LWLM to HMM's when we discuss inference (Section 2.4) and training (Section 2.5).

\subsection{Smoothing}

Because of the sparse nature of n-grams, smoothing methods have always played a central role in language modeling (Chen and Goodman, 1996). In this section we discuss the smoothing methods used to compute the two probability distributions in the LWLM: $P\left(h_{i} \mid \mathbf{h}_{i-n+1}^{i-1}, C, \gamma\right)$, the probability of the current hidden word given the $n-1$ previous hidden words and $P\left(w_{i} \mid h_{i}, C, \gamma\right)$, the probability of the observed word given the hidden word at that position. A popular method for smoothing probabilistic models is to use a Dirichlet prior (e.g. Blei et al., 2003; Goldwater and Griffiths, 2007). However, research has shown that the use of a Dirichlet prior results in suboptimal language models (MacKay and Peto, 1995; Goodman, 2001). Here, we use Kneser-Ney smoothing, of which the traditional formulation is discussed in Section 2.3.1, and a novel variant that can be used in probabilistic models is discussed in Section 2.3.2.

\subsubsection{Kneser-Ney smoothing}

In the LWLM we use Kneser-Ney smoothing, since this smoothing method was shown to consistently outperform other smoothing methods (Chen and Goodman, 1996; Goodman, 2001). Recent work has shown that this smoothing method is related to a hierarchical Pitman-Yor process (Teh, 2006). The key observation in Kneser-Ney smoothing is that not all counts collected from a training corpus should be considered equally reliable. Consider the sequence of words "Garry Tucker said". In the 5 million words Reuters corpus (see Section 2.7) this phrase is observed one time. The maximum likelihood estimate (i.e. $1 / 10^{5}$ ) is however most likely a serious overestimation, since most likely we will not observe this phrase 10 times in a corpus of 50 million words.

Kneser-Ney smoothing compensates for this overestimation by discounting the absolute counts $c\left(\mathbf{w}_{i-n+1}^{i}\right)$ with some factor $d_{n}\left(c\left(\mathbf{w}_{i-n+1}^{i}\right)\right)$ that depends on the value of the counts. The Kneser-Ney smoothing method is given by

$$
P_{a k n}\left(w_{i} \mid \mathbf{w}_{i-n+1}^{i-1}\right)=\frac{c\left(\mathbf{w}_{i-n+1}^{i}\right)-d_{n}\left(c\left(\mathbf{w}_{i-n+1}^{i}\right)\right)}{c\left(\mathbf{w}_{i-n+1}^{i-1}\right)}+\delta\left(\mathbf{w}_{i-n+1}^{i-1}\right) P_{a k n}\left(w_{i} \mid \mathbf{w}_{i-n+2}^{i-1}\right)
$$

where $d_{n}\left(c\left(\mathbf{w}_{i-n+1}^{i-1}\right)\right)$ is the discount factor for count $c\left(\mathbf{w}_{i-n+1}^{i-1}\right)$ and $\delta\left(\mathbf{w}_{i-n+1}^{i-1}\right)$ is an interpolation factor that combines the $\mathrm{n}$-gram distribution with the lower order $(n-1)$-gram distribution and is defined by

$$
\delta\left(\mathbf{w}_{i-n+1}^{i-1}\right)=1-\sum_{w_{i}} \frac{c\left(\mathbf{w}_{i-n+1}^{i}\right)-d_{n}\left(c\left(\mathbf{w}_{i-n+1}^{i}\right)\right)}{c\left(\mathbf{w}_{i-n+1}^{i-1}\right)}
$$

Eq. (1) is a recursive method, where specific, but sparse and thus possibly unreliable, higher order $n$-grams are combined with less specific, but more reliable, lower order $n$-grams. The probability distribution of bigrams and unigrams is computed in a special way. The bigram probability is computed as $P_{a k n}\left(w_{i} \mid w_{i-1}\right)=\left[\pi\left(w_{i-1} w_{i}\right)-\right.$ $\left.d_{2}\left(\pi\left(w_{i-1} w_{i}\right)\right)\right] /\left(\sum_{w_{j}} \pi\left(w_{i-1} w_{j}\right)\right)+\delta\left(w_{i-1}\right) P_{a k n}\left(w_{i}\right)$, where $\pi\left(w_{i-1} w_{i}\right)=\left|\left\{v \mid c\left(v w_{i-1} w_{i}\right)>0\right\}\right|$ is the number of different words $v$ such that the sequence $v w_{i-1} w_{i}$ occurs at least once in the training set, and the unigram probability is computed as $P_{a k n}\left(w_{i}\right)=\left(\pi\left(w_{i}\right)\right) / \sum_{w_{j}} \pi\left(w_{j}\right)$ where $\pi\left(w_{i}\right)=\mid\left\{v \mid c\left(v w_{i}\right)>0\right\}$ is the number of different words $v$ such that the sequence $v w_{i}$ occurs at least once. These special formulations of the bigram and unigram distributions are motivated by the observation that some words (e.g. "Francisco") occur frequently in a corpus, but only in very specific contexts (e.g. "San Francisco"), and that by counting the number of unique contexts of these words, we get a better estimate of the true probability of observing this word in a new context. The factors $d_{n}$ are constants selected 
to optimize the perplexity of this model on a held-out corpus. A common choice for the discount factor is (Chen and Goodman, 1996):

$$
d_{n}\left(c\left(\mathbf{w}_{i-n}^{i}\right)\right)= \begin{cases}0 & \text { if } c\left(\mathbf{w}_{i-n}^{i}\right)=0 \\ d_{n 1} & \text { if } c\left(\mathbf{w}_{i-n}^{i}\right)=1 \\ d_{n 2} & \text { if } c\left(\mathbf{w}_{i-n}^{i}\right)=2 \\ d_{n 3+} & \text { otherwise }\end{cases}
$$

where $d_{n 1}, d_{n 2}$ and $d_{n 3+}$ are constants optimized on a held-out corpus.

\subsubsection{Relative discounted Kneser-Ney smoothing}

One disadvantage of interpolated Kneser-Ney smoothing is that it is only defined for discrete counts. In the following sections we will use it in the EM-algorithm to compute $P\left(h_{i} \mid \mathbf{h}_{i-n+1}^{i-1}, C, \gamma\right)$, where we need to discount non-discrete soft counts. It is also unclear how to count the number of unique contexts of a particular word in this setting. For these reasons we propose a novel modification to the Kneser-Ney smoothing method, termed relative discounted Kneser-Ney smoothing (RDKN).

RDKN uses a relative discount factor $d_{n}\left(c\left(\mathbf{h}_{i-n+1}^{i}\right)\right)$ between 0 and 1 . The smoothing method is defined as

$$
P_{r k n}\left(h_{i} \mid \mathbf{h}_{i-n+1}^{i-1}, C, \gamma\right)=\frac{c\left(\mathbf{h}_{i-n+1}^{i}\right) \times d_{n}\left(c\left(\mathbf{h}_{i-n+1}^{i}\right)\right)}{c\left(\mathbf{h}_{i-n+1}^{i-1}\right)}+\delta\left(\mathbf{h}_{i-n+1}^{i-1}\right) P_{r k n}\left(h_{i} \mid \mathbf{h}_{i-n+2}^{i-1}\right)
$$

where the recursion ends with the unigram probability $P_{r k n}\left(h_{i}\right)=\frac{c\left(h_{i}\right)}{N_{t}}$. Similarly as before the interpolation factor $\delta\left(\mathbf{h}_{i-n+1}^{i-1}\right)$ is defined as

$$
\delta\left(\mathbf{h}_{i-n+1}^{i-1}\right)=1-\sum_{w_{i}} \frac{c\left(\mathbf{h}_{i-n+1}^{i}\right) \times d_{n}\left(c\left(\mathbf{h}_{i-n+1}^{i}\right)\right)}{c\left(\mathbf{h}_{i-n+1}^{i-1}\right)}
$$

A number of definitions could be devised for discount factor $d_{n}\left(c\left(\mathbf{h}_{i-n+1}^{i}\right)\right)$. We define $d_{n}\left(c\left(\mathbf{h}_{i-n+1}^{i}\right)\right)$ by dividing the space of counts $c\left(\mathbf{h}_{i-n+1}^{i}\right)$ into $S$ equally populated intervals with borders $c_{1}, \ldots, c_{S-1}$. Every interval is assigned a fixed discount factor $d_{s}$, defining the function $d_{n}\left(c\left(\mathbf{h}_{i-n+1}^{i}\right)\right)$ as

$$
d_{n}\left(c\left(\mathbf{h}_{i-n+1}^{i}\right)\right)= \begin{cases}d_{n 1} & \text { if } c\left(\mathbf{h}_{i-n+1}^{i}\right) \leq c_{1} \\ d_{n 2} & \text { if } c_{1}<c\left(\mathbf{h}_{i-n+1}^{i}\right) \leq c_{2} \\ \ldots & \ldots \\ d_{n S} & \text { if } c_{S-1}<c\left(\mathbf{h}_{i-n+1}^{i}\right)\end{cases}
$$

The values $0 \leq d_{n i} \leq 1$ can be optimized on a held-out corpus.

This smoothing method is different to absolute discounted Kneser-Ney smoothing in two aspects. The first difference is that we use a relative discount factor and not an absolute discount factor. The second difference is that we do not use special counts for bigram and unigram distributions, but use the relative discounted counts as given by equation 3 for the bigram distribution and use the maximum likelihood estimate for the unigram distribution. This form of Kneser-Ney smoothing can be used conveniently with soft counts (e.g. in the EM-algorithm), and in fact we see in Section 2.7 that it also outperforms Kneser-Ney smoothing when used with discrete counts.

We have described RDKN here to compute $P\left(h_{i} \mid \mathbf{h}_{i-n+1}^{i-1}, C, \gamma\right)$, but we also use it in an equivalent way to compute other probability distributions, e.g. $P\left(w_{i} \mid \mathbf{w}_{i-n+1}^{i-1}, C, \gamma\right)$, or $P\left(w_{i} \mid h_{i}, C, \gamma\right)$. The formulation of $P\left(w_{i} \mid \mathbf{w}_{i-n+1}^{i-1}, C, \gamma\right)$ can be easily derived by replacing the hidden word(s) in Eqs. (3) and (4) by observed words. RDKN smoothing for the distribution $P\left(w_{i} \mid h_{i}, C, \gamma\right)$ is given by:

$$
P\left(w_{i} \mid h_{i}, C, \gamma\right)=\frac{c\left(w_{i}, h_{i}\right) \times d\left(c\left(w_{i}, h_{i}\right)\right)}{c\left(h_{i}\right)}+\delta\left(h_{i}\right) \frac{c\left(w_{i}\right)}{N_{t}}
$$




\subsection{Inference}

During inference, we compute the expected value of the hidden words $\mathbf{h}=\left[h_{1} \ldots h_{N_{t}}\right]$ given the sequence of observed words $\mathbf{w}=\left[w_{1} \ldots w_{N_{t}}\right]$. We will see that the method traditionally used for inference in HMM's, the forward-backward algorithm, has an unfeasibly high time complexity when applied to the LWLM (Section 2.4.1). We therefore consider approximate inference, where we compute an approximation of the expected value in a dramatically lower time complexity. We develop two novel approximate inference algorithms for the LWLM, the forward-forward beam search (Section 2.4.2) and a method based on Gibbs sampling (Section 2.4.3).

\subsubsection{Forward-backward algorithm}

We first discuss the standard method used to compute the expected value of the hidden variables in HMM's, the forward-backward algorithm. This algorithm computes the probability of $h_{i}$ on position $i$ given an observed text $\mathbf{w}$ as

$$
P\left(h_{i} \mid \mathbf{w}, C, \gamma\right)=\sum_{\mathbf{h}_{i-n+2}^{i-1}} \frac{\alpha\left(\mathbf{h}_{i-n+2}^{i}\right) \beta\left(\mathbf{h}_{i-n+2}^{i}\right)}{P(\mathbf{w} \mid C, \gamma)}
$$

where $\alpha\left(\mathbf{h}_{i-n+2}^{i}\right)=P\left(\mathbf{w}_{1}^{i}, \mathbf{h}_{i-n+2}^{i}, C, \gamma\right)$ is the joint probability of observing $\mathbf{w}_{1}^{i}$ together with the sequence $\mathbf{h}_{i-n+2}^{i}$, and $\beta\left(\mathbf{h}_{i-n+2}^{i}\right)=P\left(\mathbf{w}_{i+1}^{N_{t}} \mid \mathbf{h}_{i-n+2}^{i}, C, \gamma\right)$ represents the conditional probability of observing $\mathbf{w}_{i+1}^{N_{t}}$ given the sequence $\mathbf{h}_{i-n+2}^{i}$. Both values are defined recursively as

$$
\alpha\left(\mathbf{h}_{i-n+2}^{i}\right)=P\left(w_{i} \mid h_{i}, C, \gamma\right) \sum_{h_{i-n+1}} \alpha\left(\mathbf{h}_{i-n+1}^{i-1}\right) P\left(h_{i} \mid \mathbf{h}_{i-n+1}^{i-1}, C, \gamma\right)
$$

and

$$
\beta\left(\mathbf{h}_{i-n+2}^{i}\right)=\sum_{h_{i+1}} \beta\left(\mathbf{h}_{i-n+3}^{i+1}\right) P\left(w_{i+1} \mid h_{i+1}, C, \gamma\right) P\left(h_{i+1} \mid \mathbf{h}_{i-n+2}^{i} C, \gamma\right)
$$

The values $\alpha\left(\mathbf{h}_{i-n+2}^{i}\right)$ can been seen as messages that travel from the start of the sequence to the end, while the values $\beta\left(\mathbf{h}_{i-n+2}^{i}\right)$ can be seen as messages that travel from the end of the sequence to the start. As the forward and backward messages travel through the chain, at every position in the sequence we need to store the values of $\alpha\left(\mathbf{h}_{i-n+2}^{i}\right)$ and $\beta\left(\mathbf{h}_{i-n+2}^{i}\right)$ for every possible sequence $\mathbf{h}_{i-n+2}^{i}$, requiring the storage of $2 \times|H|^{n-1}$ values, where $|H|$ is the number of hidden states. To compute the next $\alpha\left(\mathbf{h}_{i-n+3}^{i+1}\right)$ or $\beta\left(\mathbf{h}_{i-n+1}^{i-1}\right)$, we need to multiply and sum these values for every possible hidden state in the next position, requiring a computational cost of $O\left(|H|^{n}\right)$. This needs to be performed for every position, resulting in a total complexity of $O\left(|H|^{n} \times N_{t}\right)$. For more information on the forward-backward algorithm we refer to Bishop (2006).

The computational cost of the forward-backward algorithm for standard HMM's is low because most implementations of hidden Markov models use only the previous context, i.e. $n=2$, and use a small number of hidden states, i.e. $1<|H|<100$. In our model the number of the hidden variables is the size of the vocabulary, which is $10^{4}<|V|<10^{6}$ for any reasonable sized corpus, and we want to use a much larger context, e.g. $n=5$. It is thus clear that using the traditional forward-backward algorithm is not an option here.

We turn to algorithms that compute an approximation of the expected value of the hidden variables and have a lower time complexity. Work on this topic is already performed by Mitchell et al. (1995) and Yu and Kobayashi (2003), who design algorithms that reduce the time complexity of second order explicit-duration HMM's, but the suggested algorithms are still quadratic in the number of hidden states, which is also the case for the on-line learning algorithm proposed by Krishnamurthy and Moore (1993). Shue and Dey (2002) develop a efficient algorithm for HMM's that have hidden states that are nearly completely decomposable, where the hidden states can be grouped together in "super-states", which is not the case here.

Speech recognition systems often use HMM's to model the sequential dependency of (sub)phonemes. Especially when using so-called triphones (combinations of three sequential (sub)phonemes), these systems face hidden variables that can take more then $10^{3}$ different values. This is solved by clustering triphones that are similar and/or that occur infrequently in the training corpus (Woodland et al., 1994). When speech recognition systems search the most likely sequence of words given the spectral features (i.e. "decoding") they also face a search space with a size that is potentially 
exponential in the size of the vocabulary. This problem is usually solved with a Viterbi search where the least likely sequences of words are discarded (i.e. beam search) (Murveit et al., 1994; Jurafsky and Martin, 2008). This procedure is related to the forward-forward beam search presented in the next section, although it only computes the most likely sequence of words, and does not compute the probability of a word at a certain position. It can thus not be used to train a HMM.

\subsubsection{Forward-forward beam search}

In this section we develop an approximate version of the forward-backward algorithm, termed the forward-forward beam search. This algorithm approximates the expected value of the hidden variables $\mathbf{h}=\left[h_{1} \ldots h_{N_{t}}\right]$ given the observed words $\mathbf{w}=\left[w_{1} \ldots w_{N_{t}}\right]$ and the parameters $C$ and $\gamma$ using an algorithm with a time complexity dramatically lower then the original forward-backward algorithm. The algorithm is based on the observation that on every position, many hidden variables are very unlikely. By excluding these variables, we can dramatically reduce the space and time complexity, while computing the expected value up to a reasonable accuracy. We introduce a function $\operatorname{trim}\left(\alpha\left(\mathbf{h}_{i-n+2}^{i}\right)\right)$ defined by

$$
\operatorname{trim}\left(\alpha\left(\mathbf{h}_{i-n+2}^{i}\right)\right)= \begin{cases}\alpha\left(\mathbf{h}_{i-n+2}^{i}\right) & \text { if } \operatorname{rank}\left(\alpha\left(\mathbf{h}_{i-n+2}^{i}\right)\right) \geq b \\ 0 & \text { otherwise }\end{cases}
$$

where the rank $\operatorname{rank}\left(\alpha\left(\mathbf{h}_{i-n+2}^{i}\right)\right)$ is found by sorting all possible sequences $\mathbf{h}_{i-n+2}^{i}$ in descending order according to the value of $\alpha\left(\mathbf{h}_{i-n+2}^{i}\right)$. The trim function thus removes for a certain position all values that are not among the $b$ most likely values. We then define the approximate $\alpha^{\prime}\left(\mathbf{h}_{i-n+2}^{i}\right)^{3}$

$$
\alpha^{\prime}\left(\mathbf{h}_{i-n+2}^{i}\right)=\sigma_{i-n+1} P\left(w_{i} \mid h_{i}\right) \sum_{h_{i-n+1}} \operatorname{trim}\left(\alpha^{\prime}\left(\mathbf{h}_{i-n+1}^{i-1}\right)\right) P\left(h_{i} \mid \mathbf{h}_{i-n+1}^{i-1}\right)
$$

This value is an approximation of $\alpha\left(\mathbf{h}_{i-n+2}^{i}\right)$ that takes into account only the $b$ most likely values of $\alpha^{\prime}\left(\mathbf{h}_{i-n+1}^{i-1}\right)$. This value will be closer to $\alpha\left(\mathbf{h}_{i-n+2}^{i}\right)$ with increasing $b$, and will be equal if $b \geq|H|^{n-1}$. The factor $\sigma_{i-n+1}$ is the inverse of the probability mass not discarded on position $i-n+2$, given by

$$
\sigma_{i-n+1}=\frac{\sum_{\mathbf{h}_{i-n+2}^{i}} \alpha^{\prime}\left(\mathbf{h}_{i-n+1}^{i-1}\right)}{\sum_{\mathbf{h}_{i-n+2}^{i}} \operatorname{trim}\left(\alpha^{\prime}\left(\mathbf{h}_{i-n+1}^{i-1}\right)\right)}
$$

This factor makes sure that the total probability mass in the network remains equal to 1.0. On every position we need to perform the summation in Eq. (9) with a time complexity of $O(b|V|)$, and we need to sort these values to find the $b$ most likely values in the next iteration, with complexity $O(b|V| \log (b|V|))$.

Algorithm 1 shows a pseudo-code implementation of the forward beam search. When this algorithm has been executed for a given text $\mathbf{w}_{\text {train }}$, the collection $A_{i}$ contains all $\alpha^{\prime}\left(\mathbf{h}_{i-n+2}^{i}\right)$ for position $i$. For brevity we do not show the implementation of the function $\operatorname{trim}\left(A_{i}\right)$ in Algorithm 1, but this is simply the $\operatorname{trim}\left(\alpha^{\prime}\left(\mathbf{h}_{i-n+1}^{i-1}\right)\right)$ operator introduced earlier, i.e. it sorts all the values according to decreasing $\alpha$, selects the $b$ most likely one and rescales the remaining values with $\sigma_{i-n+1}$ to make sure that the total probability mass remains 1.0.

Algorithm 1. Forward beam search for text $\mathbf{w}_{N_{t}}$ to compute an approximation of the forward values $\alpha^{\prime}\left(\mathbf{h}_{i-n+2}^{i}\right)$ for every position $1 \leq i \leq N_{t}$.

\footnotetext{
${ }^{3}$ For brevity we omit the parameters $C$ and $\gamma$ from these and the following equations.
} 


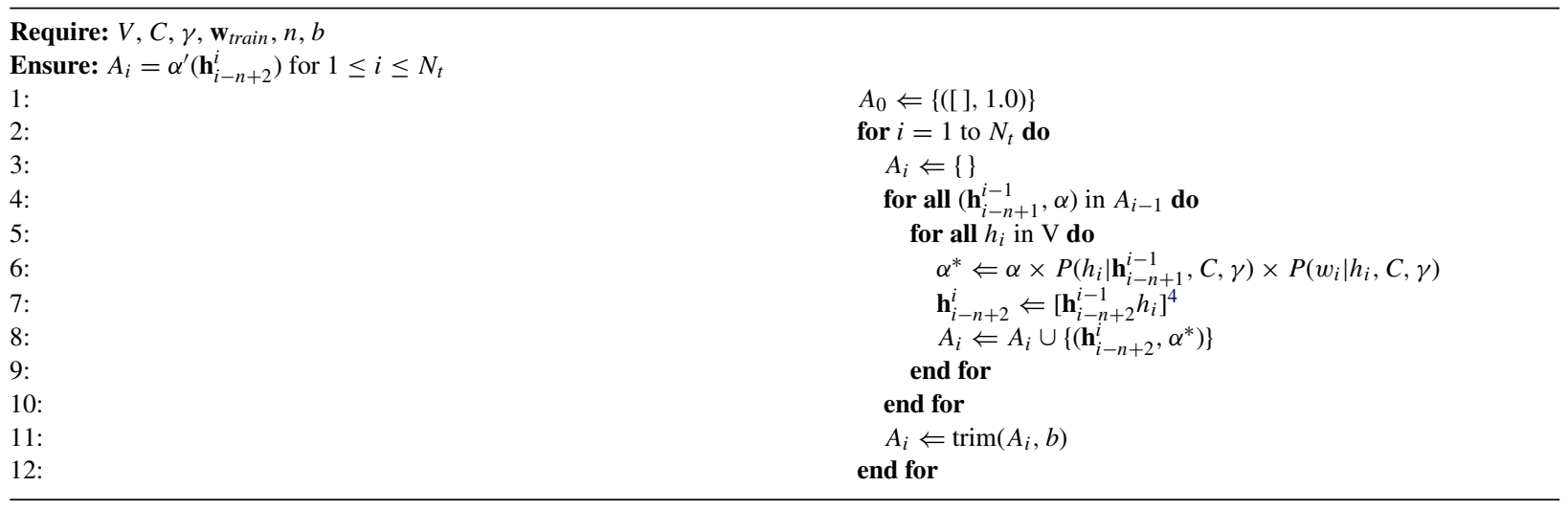

We now turn to an approximation of the backward messages. We could define a similar trimmed version of $\beta\left(\mathbf{h}_{i-n+2}^{i}\right)$, but here we face an additional difficulty. We use relative discounted Kneser-Ney smoothing to compute $P\left(h_{i+1} \mid \mathbf{h}_{i-n+2}^{i}, C, \gamma\right)$, which requires the computation of $\delta\left(\mathbf{h}_{i-n+2}^{i}\right)$. This value depends on all possible values for $h_{i+1}$, and thus requires $O(|V|)$ operations to compute. We need to compute all $\beta\left(\mathbf{h}_{i-n+2}^{i}\right)$ on a certain position before we can disregard the least likely values. We thus need to compute $P\left(h_{i+1} \mid \mathbf{h}_{i-n+2}^{i}, C, \gamma\right)$ for all possible values of $h_{i-n+2}$, and the total number of operations required at every position would be $O\left(|V|^{2}\right)$. When faced with a large vocabulary this time complexity becomes too high, and we replace the backward step with a number of forward steps.

We introduce a new forward probability $\gamma\left(\mathbf{h}_{i-n+2}^{i}, h_{j}\right)$ which is the joint probability of observing the words $\mathbf{w}_{1}^{i}$, the sequence $\mathbf{h}_{i-n+2}^{i}$ and the hidden variable $h_{j}$. This value is defined for $i \geq j$ and is given by

$$
\gamma\left(\mathbf{h}_{i-n+2}^{i}, h_{j}\right)= \begin{cases}P\left(w_{i} \mid h_{i}\right) \sum_{h_{i-n+1}} \alpha\left(\mathbf{h}_{i-n+1}^{i-1}\right) P\left(h_{i} \mid \mathbf{h}_{i-n+1}^{i-1}\right) & \text { if } i=j \\ P\left(w_{i} \mid h_{i}\right) \sum_{h_{i-n+1}} \gamma\left(\mathbf{h}_{i-n+1}^{i-1}, h_{j}\right) P\left(h_{i} \mid \mathbf{h}_{i-n+1}^{i-1}\right) & \text { if } i>j\end{cases}
$$

This value is similar to $\alpha\left(\mathbf{h}_{i-n+2}^{i}\right)$, but also includes the probability of generating $h_{j}$ at position $j$. We can interpret $\gamma\left(\mathbf{h}_{i-n+2}^{i}, h_{j}\right)$ as a series of messages, where for every different value of $h_{j}$, a series of messages $\gamma\left(\mathbf{h}_{i-n+2}^{i}, h_{j}\right)$ is passed from position $j$ to the end of the sequence. A trimmed version of this variable is defined as

$$
\gamma^{\prime}\left(\mathbf{h}_{i-n+2}^{i}, h_{j}\right)= \begin{cases}\sigma_{i-n+1} P\left(w_{i} \mid h_{i}\right) \sum_{h_{i-n+1}} \operatorname{trim}\left(\alpha^{\prime}\left(\mathbf{h}_{i-n+1}^{i-1}\right)\right) P\left(h_{i} \mid \mathbf{h}_{i-n+1}^{i-1}\right) & \text { if } i=j \\ \sigma_{i-n+1} P\left(w_{i} \mid h_{i}\right) \sum_{h_{i-n+1}} \operatorname{trim}\left(\gamma^{\prime}\left(\mathbf{h}_{i-n+1}^{i-1}, h_{j}\right)\right) P\left(h_{i} \mid \mathbf{h}_{i-n+1}^{i-1}\right) & \text { if } i>j\end{cases}
$$

The sum in this equation has time complexity $O(b|V|)$ and sorting the values has complexity $O(b|V| \log (b|V|))$. Doing so for every position in the sequence results in a time complexity of $O\left(N_{u} \times[b|V|+b|V| \log (b|V|)]\right)$. After passing all messages to the end of the sequence, we have a collection of messages $\gamma^{\prime}\left(\mathbf{h}_{N_{u}-n+2}^{N_{u}}, h_{j}\right)$. To compute the probability of $h_{j}$ given $\mathbf{w}_{\text {test }}$ we sum over all possible values of $\mathbf{h}_{N_{u}-n+2}^{N_{u}}$, i.e.

$$
P\left(h_{j} \mid \mathbf{w}_{\text {test }}, C\right)=\frac{\sum_{\mathbf{h}_{N_{u}-n+2}^{N_{u}}} \gamma\left(\mathbf{h}_{N_{u}-n+2}^{N_{u}}, h_{j}\right)}{P\left(\mathbf{w}_{\text {test }} \mid C\right)} \simeq \frac{\sum_{\mathbf{h}_{N_{u}-n+2}^{N_{u}}} \gamma^{\prime}\left(\mathbf{h}_{N_{u}-n+2}^{N_{u}}, h_{j}\right)}{P\left(\mathbf{w}_{\text {test }} \mid C\right)}
$$

\footnotetext{
${ }^{4}$ We use $\left[\mathbf{h}_{i-n+2}^{i-1} h_{i}\right]$ as a shorthand for the sequence obtained by appending the hidden word $h_{i}$ to the sequence $\mathbf{h}_{i-n+2}^{i-1}$.
} 
Summarizing, we first pass a series of forward messages $\alpha\left(\mathbf{h}_{i-n+1}^{i-1}\right)$ from the start of the sequence to the end. We then pass for every position $j$ in the sequence, a series of $\gamma\left(\mathbf{h}_{i-n+1}^{i-1}, h_{j}\right)$ messages from that position to the end of the sequence.

One disadvantage of this approach is that in the original forward-backward algorithm only a single pass of forward messages and a single pass of backward messages is required to compute the probability distribution for every $h_{i}$. In our formulation however we first perform a single pass of forward messages, and then perform, for every position $j$, a pass of forward messages $\gamma\left(\mathbf{h}_{i-n+2}^{i}, h_{j}\right)$ from position $j$ to the end of the sequence, which increases the computational the complexity of the algorithm. Therefore we make an additional assumption: we assume that the probability distribution of $h_{j}$ given $\mathbf{w}_{1}^{N_{t}}$ is approximately equal to the probability of $h_{i}$ given the words $\mathbf{w}_{1}^{j+\delta}$ for a certain distance $\delta$ :

$$
P\left(h_{j} \mid \mathbf{w}_{1}^{N_{t}}, C^{\tau}\right) \simeq P\left(h_{j} \mid \mathbf{w}_{1}^{j+\delta}, C^{\tau}\right)
$$

This is plausible since the words that occur in the sequence far away from the hidden word will have little influence on this word. The final method to compute the probability of $h_{j}$ is then

$$
P\left(h_{j} \mid \mathbf{w}_{\text {test }}, C^{\tau}\right) \simeq \frac{\sum_{\mathbf{h}_{j+\delta-n+2}^{j+\delta}} \gamma^{\prime}\left(\mathbf{h}_{j+\delta-n+2}^{j+\delta}, h_{j}\right)}{P\left(\mathbf{w}_{\text {test }} \mid C^{\tau}\right)}
$$

The time complexity of the forward-forward beam search is $O\left(N_{u} \times(1+\delta) \times(b|V|+b|V| \log (b|V|))\right.$, which will be, even for fairly high values for $b$ and $\delta$, much lower than the original time complexity of the forward-backward algorithm.

Algorithm 2. Forward-forward beam search to compute the expected values of $h_{1}, \ldots, h_{N_{t}}$ given text $\mathbf{w}_{N_{t}}$.

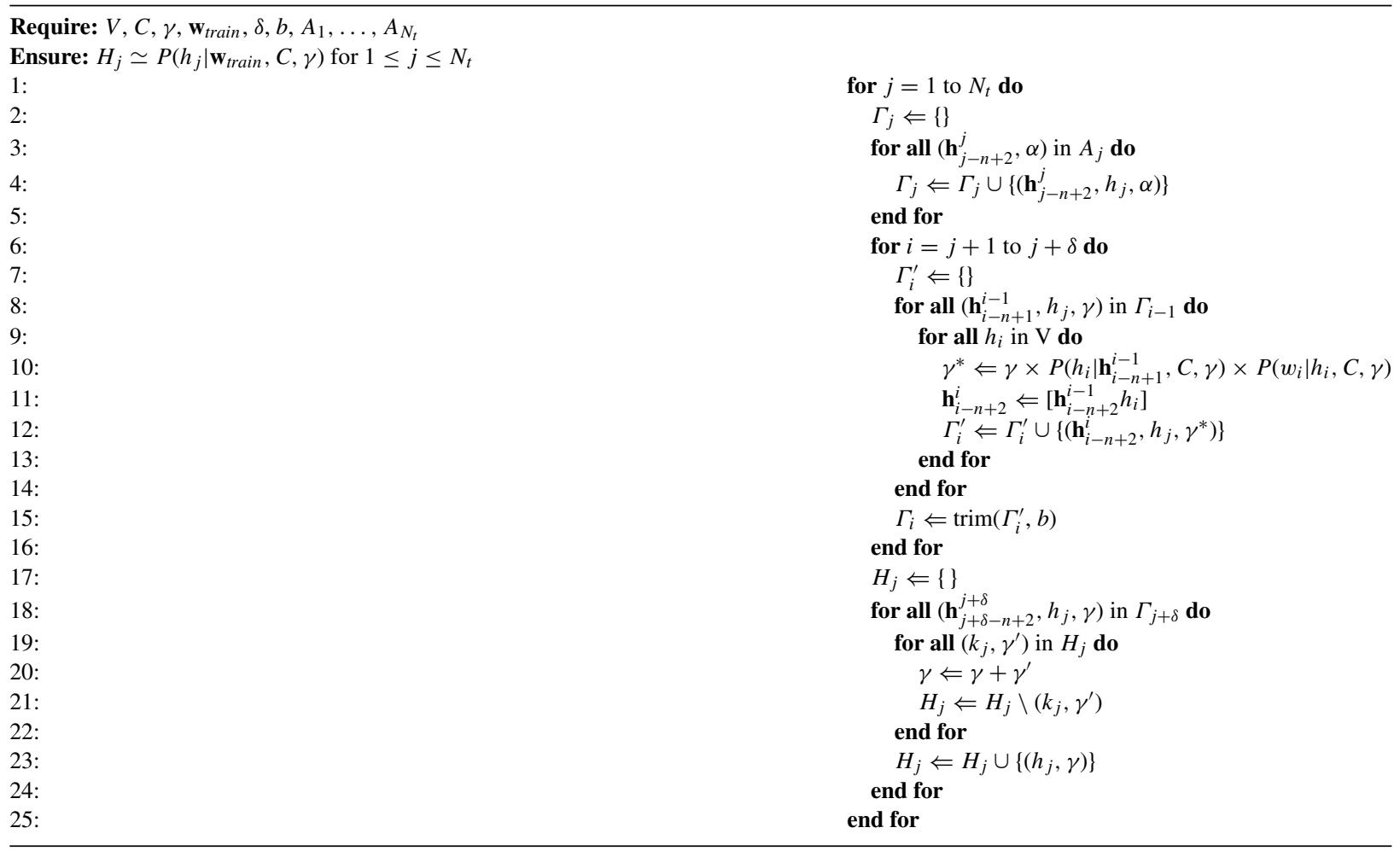

The pseudo-code for the forward-forward beam search is shown in algorithm 2 . The $\gamma^{\prime}\left(\mathbf{h}_{j-n+1}^{j-1}, h_{i}\right)$ values are stored in a data structure $\Gamma_{j}=\left\{\left(\mathbf{h}_{j-n+1}^{j-1}, h_{i}, \gamma\right)\right\}$ where $\gamma$ is the value of $\gamma^{\prime}\left(\mathbf{h}_{j-n+1}^{j-1}, h_{i}\right)$. In every step we compute for every possible $\left(\mathbf{h}_{j-n+1}^{j-1}, h_{i}, \gamma\right)$ in $\Gamma_{j-1}$, the new probability $\gamma^{*}$, and add $\left(\mathbf{h}_{j-n+2}^{j}, h_{i}, \gamma^{*}\right)$ to $\Gamma_{j}^{\prime}$. 
We have shown how we use the forward-forward beam search to compute the expected value of the hidden variable $h_{j}$ at every position $j$ given the observed words $\mathbf{w}_{\text {train }}$. However, sometimes (e.g. during training) we also need to compute the expected value of sequences of hidden words $\mathbf{h}_{j-m+1}^{j}$ for $2 \leq m \leq n$. This can be done with only a small modification to the forward-forward beam search as presented here. Basically, instead of propagating $\gamma\left(\mathbf{h}_{i-n+1}^{i-1}, h_{j}\right)$ for every likely value of the hidden variable $h_{j}$, we propagate $\gamma\left(\mathbf{h}_{i-n+1}^{i-1}, \mathbf{h}_{j-m+1}^{j}\right)$ for every likely value of $\mathbf{h}_{j-m+1}^{j}$. This can be done with a time complexity of $O\left(N_{u} \times(1+\delta) \times(b|V|+b|V| \log (b|V|))\right.$ and thus does not change the time complexity of the full algorithm.

\subsubsection{Gibbs sampling}

Although the forward-forward beam search offers a fast method to compute the expected value of the hidden words, in some applications computational speed is less of a requirement then easiness of implementation. In this section we develop a method based on Gibbs sampling that also computes the expected value, but is significantly easier to implement. It does so by sacrificing some computational speed, although it still runs much faster then the standard forward-backward algorithm. This second method also allows us to assess the performance of the forward-forward beam search.

Gibbs sampling is a Markov Chain Monte Carlo sampling technique that iteratively generates a number of samples of the expected value of the hidden variables. In the first iteration the values of the hidden variables $\mathbf{h}^{(1)}$ are typically initialized randomly (although we will also consider a better initialization in Section 2.5). After initializing, in every iteration $\tau$ the current sample $\mathbf{h}^{(\tau)}$ is used to generate the next sample $\mathbf{h}^{(\tau+1)}$. Every position $i$ is visited in turn and the distribution of the hidden variable $h_{i}$ at that position is computed as

$$
P\left(h_{i} \mid \mathbf{h}_{-i}, \mathbf{w}, C^{(\tau)}, \gamma\right)=\frac{P\left(w_{i} \mid h_{i}, C^{(\tau)}, \gamma\right) P\left(h_{i} \mid \mathbf{h}_{i-n+1}^{i-1}, C^{(\tau)}, \gamma\right) \prod_{j=i+1}^{i+n-1} P\left(h_{j} \mid\left[\mathbf{h}_{j-n+1}^{i-1} h_{i} \mathbf{h}_{i+1}^{j-1}\right], C^{(\tau)}, \gamma\right)}{\sum_{h_{i}^{*} \in V} P\left(w_{i} \mid h_{i}^{*}, C^{(\tau)}, \gamma\right) P\left(h_{i}^{*} \mid \mathbf{h}_{i-n+1}^{i-1}, C^{(\tau)}, \gamma\right) \prod_{j=i+1}^{i+n-1} P\left(h_{j} \mid\left[\mathbf{h}_{j-n+1}^{i-1} h_{i}^{*} \mathbf{h}_{i+1}^{j-1}\right], C^{(\tau)}, \gamma\right)}
$$

where $\mathbf{h}_{-i}$ is the collection of values for all hidden variables except for $h_{i}, h_{i}^{*}$ ranges over all values in the vocabulary $V, C^{(\tau)}$ is the collection of counts derived from $\mathbf{h}_{-i}$ and $\mathbf{w}, P\left(h_{i} \mid \mathbf{h}_{i-n+1}^{i-1}, C^{(\tau)}, \gamma\right)$ is the probability of $h_{i}$ given the sequence of hidden variables $\mathbf{h}_{i-n+1}^{i-1}$, and $P\left(h_{j} \mid\left[\mathbf{h}_{j-n+1}^{i-1} h_{i} \mathbf{h}_{i+1}^{j-1}\right], C^{(\tau)}, \gamma\right)$ is the probability of $h_{j}$ given the sequence of hidden variables $\left[\mathbf{h}_{j-n+1}^{i-1} h_{i} \mathbf{h}_{i+1}^{j-1}\right]$. Note that as before we use $\left[\mathbf{h}_{j-n+1}^{i-1} h_{i} \mathbf{h}_{i+1}^{j-1}\right]$ to denote the sequence of hidden words that is obtained by appending $h_{i}$ and $h_{i+1}^{j-1}$ to $h_{j-n+1}^{i-1}$.

The probability in Eq. (10) is computed for all possible values of $h_{i}$, one value is selected according to this distribution, and the hidden variable is set to this value. Gibbs sampling then continues by sampling a value for $h_{i+1}$ and so on until a new value is sampled for all variables in $\left[h_{1} \ldots h_{N_{t}}\right]$. This process is repeated for a number of iterations. After an initial burn-in period, the samples represent true samples from the likelihood $P(\mathbf{h} \mid \mathbf{w}, \gamma)$. A number of samples can then be combined to compute an approximate distribution of the expected value of every hidden variable.

How does the Gibbs sampling method relate to the forward-forward beam search? Both methods aim at the same goal: find the expected value of the hidden words. The forward-forward beam search will find the expected value by considering only the most likely hidden variables at every position. By doing so it ignores a large part of the search space and thus achieves a significant speedup. However, a consequence of this choice is that a value that is not among the most likely values will never be considered. A good initialization is thus very important with this method (see next section). The Gibbs sampling method does not have this disadvantage, as it generates samples from the entire search space. One disadvantage however of Gibbs sampling is that for a given position, only a single value is sampled. This needs to be repeated many times to compute a reasonable approximation of the distribution for the hidden variable at that position. Furthermore, when distributions are "spiky", i.e. one value has a very high probability and other values a very low probability, even more samples are needed because the probability of sampling one of the values with low probability is very low, resulting in long computation times for Gibbs sampling (see Section 2.7). 


\subsection{Training}

HMM's are typically trained with the Baum-Welch algorithm (Baum et al., 1970), which is an instance of the expectation-maximization (EM) algorithm that optimizes the model on the training text $\mathbf{w}_{\text {train }}$. The algorithm starts from a random initialization of the counts $C^{1}$. This estimate is then improved in a number of iterations, where every iteration performs an expectation step and a maximization step. In the expectation step the expected value of the hidden variables is computed with the forward-backward algorithm. In the maximization step the new counts $C^{\tau}$ are derived from these expected values. Since every step improves the likelihood of the model on $\mathbf{w}_{\text {train }}$, the Baum-Welch algorithm is guaranteed to find a (possibly local) maximum likelihood estimate.

For the LWLM, we want to find the parameters $C$ and $\gamma$ that maximize the likelihood of the model on unseen texts. To achieve this goal, we use two corpora, a large corpus $\mathbf{w}_{\text {train }}=\left[w_{1} \ldots w_{N_{t}}\right]$, used to determine the counts $C$, and a smaller corpus $\mathbf{w}_{\text {heldout }}=\left[w_{1} \ldots w_{N_{h}}\right]$, used to determine the smoothing parameters $\gamma$. We additionally adapt the following aspects of the Baum-Welch algorithm: initialization, inference and smoothing.

\subsubsection{Initialization}

Standard Baum-Welch uses a random initialization of the hidden variables. Because of the large dimension of the hidden variables for the LWLM (the entire vocabulary $V$ ), a random initialization would result in many EM iterations, and would risk getting stuck in a local, suboptimal maximum. We initialize the values of the hidden variables by setting the value of every hidden word $h_{i}$ at position $i$ as the distribution of observed words that are likely to occur at that position, given the words occurring before and after this position. For this, we first train a standard n-gram language model with RDKN smoothing on the observed words of the training corpus, which gives the probability distribution $P\left(w_{i} \mid \mathbf{w}_{i-n+1}^{i-1}\right)$ of the current word given the $n-1$ previous words. We then set the probability distribution of every hidden word $h_{i}$ in the training corpus to $P\left(w_{i} \mid \mathbf{w}_{1}^{i-1}, \mathbf{w}_{i+1}^{N_{t}}\right)$, i.e. to the probability of the observed word, given all words that occur before and after this word. This probability is computed as

$$
P\left(w_{i} \mid \mathbf{w}_{1}^{i-1}, \mathbf{w}_{i+1}^{N_{t}}\right) \sim P\left(w_{i} \mid \mathbf{w}_{i-n+1}^{i-1}\right) \prod_{j=i+1}^{i+n-1} P\left(w_{j} \mid \mathbf{w}_{j-n+1}^{i-1} w_{i} \mathbf{w}_{i+1}^{j-1}\right)
$$

This assignment makes the assumption that likely hidden words at a certain position are the words that are likely to be observed in that context, or more specifically, that are likely to be generated by the $n-1$ previous observed words, and that are likely to generate the $n-1$ next observed words. We assign these distributions to the hidden variable $h_{i}$ on every position and then construct the initial counts $C^{1}$ from these distributions by collecting the following soft counts: the frequency of a hidden word $h_{i}$ generating an observed word $w_{i}$, and the frequency of the sequence $\mathbf{h}_{i-m+1}^{i}$ occurring in $\mathbf{h}=\left[h_{1} \ldots h_{N_{t}}\right]$, where the length $m$ ranges from $n$ to 1 .

\subsubsection{Inference}

The second modification to the Baum-Welch algorithm is the use of an approximate method to compute the value of $P\left(h_{i} \mid \mathbf{w}_{\text {train }}, C, \gamma[\tau]\right)$. As discussed in the previous sections, we hereby consider the forward-forward beam search or Gibbs sampling. The approximate estimates found by one of this methods are used to construct the new counts $C^{\tau+1}$.

\subsubsection{Smoothing}

The third modification is that we also update the smoothing parameters $\gamma^{\tau+1}$ : we use an iterative line-search to find the parameters that optimize the likelihood $\mathcal{L}\left(C, \gamma[\tau+1] ; \mathbf{w}_{\text {heldout }}\right)$ of the parameters given the held-out corpus. After the smoothing parameters have been optimized, we again perform an iteration of the EM-algorithm. This cycle is repeated until the parameters have converged and the perplexity on the held-out corpus does not decrease anymore. 


\subsection{Computing the probability of an unseen text}

We have explained how we can estimate the hidden words for a particular given text. However, we would also like to use the model to predict the probability $P\left(\mathbf{w}_{\text {test }} \mid C, \gamma\right)$ of an unseen text $\mathbf{w}_{\text {test }}=\left[w_{1} \ldots w_{N_{u}}\right]$. Standard HMM's use the forward algorithm to compute this value

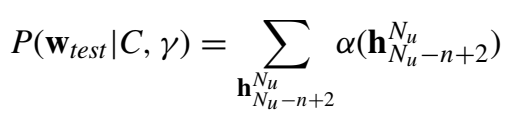

where $\alpha\left(\mathbf{h}_{i-n+2}^{i}\right)$ is defined as in the previous section. This algorithm thus passes a series of messages from the start of the sequence to the end. The messages $\alpha\left(\mathbf{h}_{N_{u}-n+2}^{N_{u}}\right)$ at the end of the sequence are then summed, resulting in the probability of observing the entire sequence.

Since the time complexity of this algorithm is $O\left(N_{u}|V|^{n}\right)$, we use the forward-forward beam search. This method uses the trimmed $\alpha^{\prime}\left(\mathbf{h}_{N_{u}-n+2}^{N_{u}}\right)$ to compute

$$
P\left(\mathbf{w}_{\text {test }} \mid C, \gamma\right)=\sum_{\mathbf{h}_{N_{u}-n+2}^{N_{u}}} \alpha^{\prime}\left(\mathbf{h}_{N_{u}-n+2}^{N_{u}}\right)
$$

where $\alpha^{\prime}\left(\mathbf{h}_{N_{u}-n+2}^{N_{u}}\right)$ is defined as in the previous section. Note that, although this method drops unlikely $\alpha^{\prime}\left(\mathbf{h}_{i-n+2}^{i}\right)$ values, it assigns a non-zero probability to every possible sequence of observed words, thanks to the smoothing in Eq. (5). This method is outlined in Algorithm 3. It is important to note that this method is used regardless of the method used to train the LWLM (i.e. forward-forward beam search or Gibbs sampling). Gibbs sampling cannot be used to estimate the probability of an unseen text, since it only computes samples from $P(\mathbf{h} \mid \mathbf{w}, C, \gamma)$, but never computes $P\left(\mathbf{w}_{\text {test }} \mid C, \gamma\right)$.

Algorithm 3. Compute probability of observed text $\mathbf{w}_{\text {test }}$.

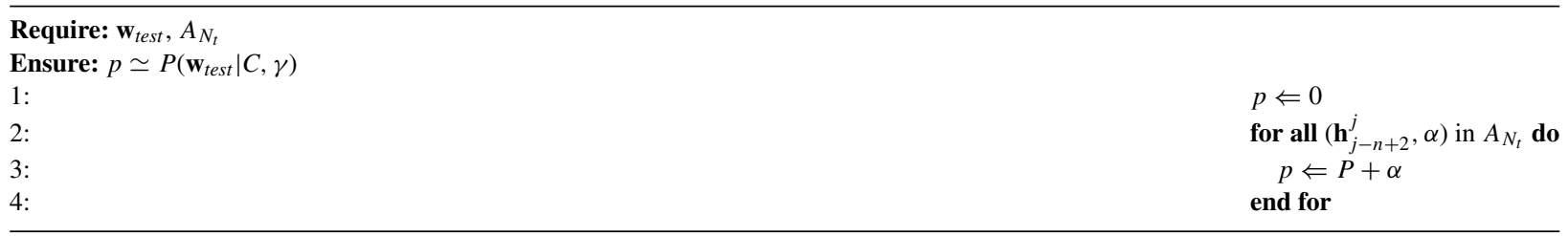

n-Gram models that map words to hidden variables or clusters often improve the ability of a model to predict the probability of unseen sequences, but they can also hurt precision when assigning too much weight to unseen sequences (Goodman, 2001). For this reason it is often a good idea to interpolate these models with a standard n-gram model. Given a value of the hidden variable $h_{i}$, the probability of the observed word is given by

$$
P\left(w_{i} \mid h_{i}, \mathbf{w}_{i-n+1}^{i-1}, C, \gamma\right)=(1-\epsilon) P\left(w_{i} \mid h_{i}, C, \gamma\right)+\epsilon P\left(w_{i} \mid \mathbf{w}_{i-n+1}^{i-1}, C, \gamma\right)
$$

where $0 \leq \epsilon \leq 1$ is a constant value optimized on a held-out text and $P\left(w_{i} \mid w_{i-n+1}^{i-1}, C, \gamma\right)$ is the probability of $w_{i}$ given the $n-1$ previous observed words using a standard n-gram language model on the observed words, smoothed with relative discounted Kneser-Ney smoothing. We will see in Section 2.7.2 that this combination achieves better results then either model alone, especially for small values of $\epsilon$.

\subsection{Evaluating the LWLM}

In this section we evaluate the performance of the LWLM. The performance of a language model is typically measured in terms of the perplexity of the model on the unseen test corpus $\mathbf{w}_{t e s t}=\left[w_{1} \ldots w_{N_{u}}\right]$ of length $N_{u}$

$$
\sqrt[N_{u}]{\frac{1}{P\left(\mathbf{w}_{\text {test }}\right)}}
$$


Table 2

Results in terms of perplexity of the 5-gram models with absolute discounted Kneser-Ney (ADKN) or relative discounted Kneser-Ney (RDKN) smoothing, of the full-IBM-predict class-based language model and of the latent words language model (LWLM) and its interpolated version (int. LWLM), trained with forward-forward beam search or with Gibbs sampling.

\begin{tabular}{lrrr}
\hline Method & Reuters & APNews & EnWiki \\
\hline ADKN & 114.96 & 134.42 & 161.41 \\
RDKN & 112.37 & 132.99 & 160.83 \\
IBM & 108.38 & 125.65 & 149.21 \\
LWLM (beam search) & 108.78 & 124.57 & 151.98 \\
int. LWLM (beam search) & $\mathbf{9 6 . 4 5}$ & 112.81 & $\mathbf{1 3 8 . 0 3}$ \\
LWLM (Gibbs sampling) & 108.86 & 124.74 & 152.83 \\
int. LWLM (Gibbs sampling) & 96.49 & $\mathbf{1 1 2 . 5 5}$ & 138.49 \\
\hline
\end{tabular}

where $P\left(\mathbf{w}_{\text {test }}\right)=\prod_{i=1}^{N_{u}} P\left(w_{i} \mid \mathbf{w}_{1}^{i-1}\right)$ is the probability of the test corpus (Jurafsky and Martin, 2008). The perplexity can be seen as the confusion of the model. For example, a language model that assigns equal probability to 100 words at every position in the held-out corpus has a perplexity of 100 (assuming that at every position the observed word belongs to the set of predicted words). One attractive property of the perplexity measure is that the "true" model for any data source will have the lowest possible perplexity for that source. Thus, the lower the perplexity of our model, the closer it is in some sense, to the model of natural language employed by humans.

We first compare the performance of the latent words language model with other language models in Section 2.7.1 and then evaluate certain aspects of the proposed models in Section 2.7.2.

\subsubsection{Comparison to existing language models}

We compare the performance of the language models presented here on three different corpora. The first two corpora, Reuters ${ }^{5}$ and APNews, ${ }^{6}$ are collections of news articles that are distributed respectively by the Reuters and Associated Press news agencies. Both corpora have a large portion of financial news, together with a smaller amount of general news. The second corpus EnWiki is a collection of encyclopedia articles from the English language Wikipedia. The length of every corpus is 5 million word tokens. The APNews corpus was preprocessed as described by Bengio et al. (2003), the other two corpora were preprocessed by concatenating all sentences and mapping all words that occurred fewer than three times in the combined training, held-out and test corpus to a new, "UNKNOWN" symbol. All punctuation was preserved. This preprocessing is common in language modeling, see e.g. Goodman (2001). It reduces the the vocabulary sizes of the corpora to 39,373 for Reuters, 15,247 for APNews and 54,371 for EnWiki. The larger vocabulary size of the EnWiki corpus reflects the larger topical variation of the EnWiki corpus. For both corpora we use a fixed section of $100 \mathrm{~K}$ consecutive words as a heldout corpus, used to optimize the smoothing parameters of every method, and a section of $100 \mathrm{~K}$ consecutive words as a test corpus, used to measure the perplexity of every model.

Table 2 shows the performance of the different models on the three corpora. We compare 5-gram models with absolute discounted Kneser-Ney smoothing $(A D K N)$, relative discounted Kneser-Ney smoothing $(R D K N)$ and of the full-IBM-predictmodel (IBM), which is an existing language model that creates clusters of words that are syntactically and semantically similar, but contrary to our method, all words are hard-assigned to a single cluster (Goodman, 2001). It was found to be the best cluster-based model by Goodman (2001). We compare these language models with the latent words language model ( $L W L M)$ and the interpolated version of this model (int. $L W L M$ ). This model was trained either with the forward-forward beam search or with Gibbs sampling. ${ }^{7}$ Note that the approximate methods used for inference do not consider all possible sequences of hidden words, but they do model every possible sequences of observed words because of the smoothing used in equation 5 .

\footnotetext{
5 See http://about.reuters.com/researchandstandards/corpus/.

6 We thank Yoshua Bengio and Hugo Larochelle for providing this corpus.

${ }^{7}$ Note that, independently of the training method, we always use the forward beam search to compute the perplexity on the test corpus.
} 

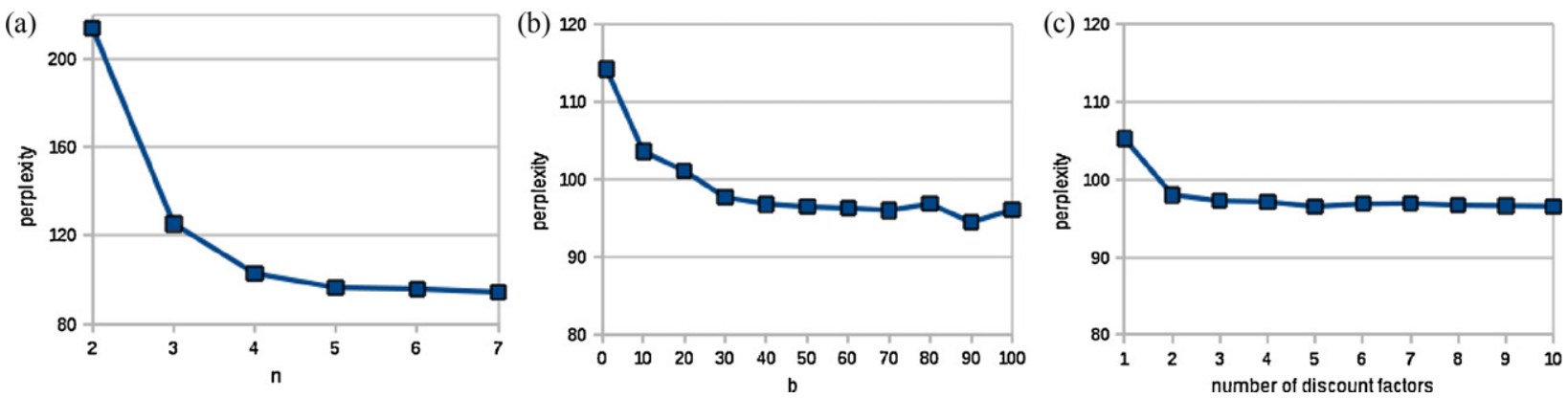

Fig. 2. Perplexity of the interpolated LWLM, where we vary the length $n$ of the n-gram (a) or the beam size $b$ (b), or the number of discount factors (c).

From Table 2 we make the following observations:

- Relative discounted Kneser-Ney smoothing consistently outperforms absolute discounted Kneser-Ney smoothing. We have performed a fair comparison where both models use the same number of discount parameters. This refutes any optimality claims that have been made for absolute discounted Kneser-Ney smoothing (Chen and Goodman, 1996; Goodman, 2001). In fact we suspect that smoothing methods that use more complex methods for discounting will prove to be even more successful.

- The latent words language model outperforms both variants of Kneser-Ney smoothing. This shows that the algorithm successfully learned word similarities that alleviated the sparseness problems of n-gram models.

- The interpolated latent words language model outperforms all other models. Compared to absolute discounted Kneser-Ney, which is a state-of-the-art baseline, the interpolated LWLM (trained with the forward-forward beam search) performs between $14 \%$ and $18 \%$ better. Compared to the full-IBM-predict model, which is to the best of our knowledge the best n-gram based language model, the model here presented performs between $7 \%$ and $10 \%$ better.

- The performance of the latent words language model is very similar when trained with Gibbs sampling or with the forward-forward beam search in terms of perplexity. The forward-forward beam search however resulted in much faster computing times, e.g. on the Reuters corpus it needed approximately $120 \mathrm{~h}$ of computing time on a $2.7 \mathrm{GHz}$ core computer, compared to approximately $800 \mathrm{~h}$ of computing time for Gibbs sampling. This shows that the forward-forward beam search is an good approximate method to use when faced with models with a large number of hidden variables.

\subsubsection{Additional experiments}

In the following paragraphs we investigate certain properties of the interpolated LWLM. For all experiments we train the model on the 5 million Reuters corpus using the forward-forward beam search, and measure the perplexity on the test section of this corpus. In a first experiment we justify the choice for a relatively large value for $n$. Fig. 2(a) shows how the perplexity of the model reduces with increasing $n$. As $n$ increases, we take into account a larger context, resulting a lower perplexity of the model. We see that we need to use a value of $n=4$ or $n=5$ for a competitive language model, justifying the approximate techniques for training and inference developed in this article. In all other experiments we have set $n=5$.

In a second experiment we see the influence of the beam size for the LWLM. For this we measure the perplexity of the interpolated LWLM with different beam sizes. We see from Fig. 2(b) that as we increase the beam size, the gain by the LWLM compared to standard RDKN (which achieves 112.37 on this corpus) becomes greater, although this effect levels off with a beam size greater then 40 . In our experiments we have chosen a conservative beam width of 50 .

In a third experiment we see the influence of the number of discount parameters employed in our model. Remember that for relative discounted Kneser-Ney smoothing (Section 2.3.2) for the n-grams $h_{i-n+1} \ldots h_{i}$ of a particular length $n$ we create for the counts $c\left(h_{i-n+1} \ldots h_{i}\right)$ a number of equally populated intervals. The counts in a particular interval are then discounted with a certain factor, unique to this interval. As we increase the number of intervals (and thus the number of discount factors), the model has more expressive power, most likely resulting in a more accurate model. Fig. 2(c) shows how the perplexity of the model changes with the number of intervals employed in the discount function. 
(a)

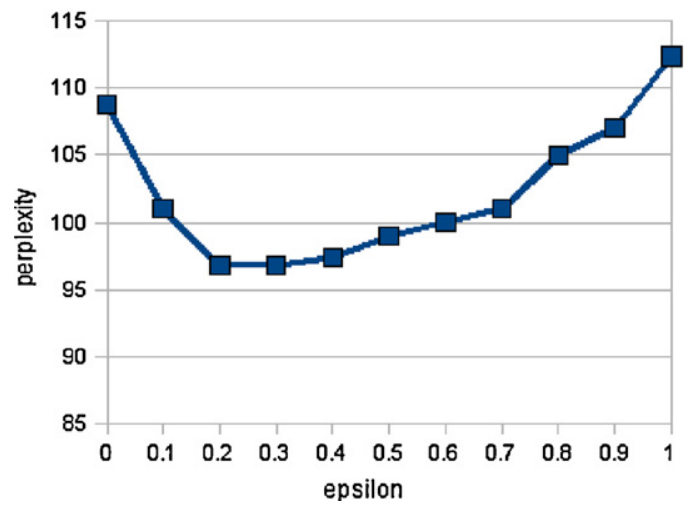

(b)

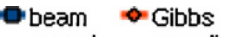

search sampling

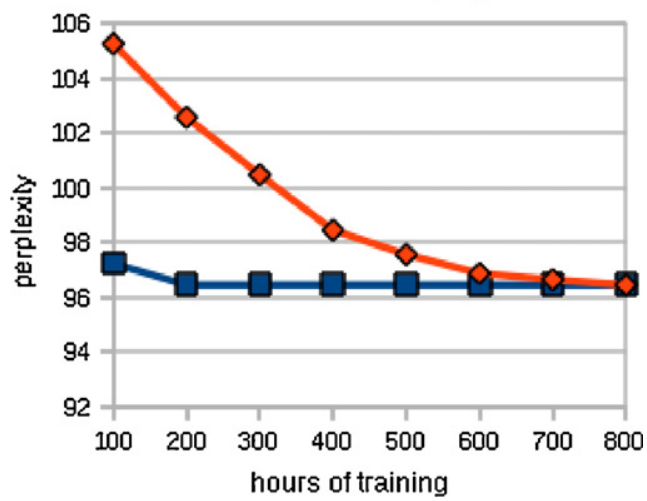

Fig. 3. The perplexity of the interpolated LWLM while varying the interpolation factor epsilon (a) and number of hours used to train with forward-forward beam search or Gibbs sampling method (b).

We see that it is important to use more then one interval, but after that performance levels off quite quickly. In all other experiments we have set the number of intervals to 5 .

In a fourth experiment we investigate the influence of the interpolation factor $\epsilon$, changing the value of $\epsilon$ from 0 to 1 . With $\epsilon=1$ the LWLM is just a standard language model on the observed words. As $\epsilon$ decreases, the influence of the latent words increases and the perplexity decreases, although it helps to assign a small weight to $\epsilon$. For every corpus the value of this variable is optimized on the heldout section of that corpus, e.g. for the Reuters corpus 0.247 was selected, which is close to the optimal value for the test section of this corpus, which is approximately 0.251 (see Fig. 3).

\section{Using the LWLM in natural language processing}

In the previous section we have shown that the hidden words in the LWLM capture semantic and syntactic similar words and that these hidden words depend on the context, essentially disambiguating the word. We have also seen how these hidden words reduce the sparseness problems of traditional n-gram models. In this section we turn to a second use of the latent words: as an extra representation of a text that can be used in natural language processing (NLP) tasks. Most supervised NLP methods face the difficulty of the large vocabulary size of any given corpus. Many words are never encountered in the annotated training corpus, and words that are encountered in the training corpus are often ambiguous. In this section we show that the hidden words learned by the LWLM can be used successfully in information extraction tasks. We do this by expanding two state-of-the-art classifiers with latent words, for word sense disambiguation (Section 3.1) and semantic role labeling (Section 3.2).

\subsection{Word sense disambiguation}

Many words are ambiguous, i.e. they can have several different meanings in a text. Word sense disambiguation (WSD) is defined as the task of selecting for every word in a text, the right sense for that word from a dictionary of senses, depending on the context the word is used in. The most frequently used dictionary for WSD is WordNet (Fellbaum, 1998), which was also used in the Senseval and Semeval workshops (Kilgarriff, 1998; Snyder and Palmer, 2004). WordNet (v. 2.1) contains the senses of more than 155,000 different words, which are organized into synsets, where every synset is a collection of words that are synonyms, or that are closely related, and that represent a single concept or entity. An example of such a synset is "person, individual, someone, somebody, mortal, soul", referring to a human being. 


\subsubsection{Discriminative WSD model}

Every word to be disambiguated is described by a feature vector $\mathbf{F t}_{i}$ that contains syntactic and semantic information on the context of that word (see next section). We model the probability $P\left(\right.$ Synset $\left._{i} \mid \mathbf{F t r}_{\mathrm{i}}\right)$ of assigning a synset Synset $_{i}$ to a given word $w_{i}$ described by features $\mathbf{F t r}_{\mathrm{i}}$ as an exponential distribution ${ }^{8}$

$$
P\left(\text { Synset }_{i} \mid \mathbf{F t r}_{\mathrm{i}}, \theta\right)=\frac{1}{Z\left(\mathbf{F t r}_{\mathrm{i}}\right)} \prod_{k} g\left(\text { Synset }_{i}, k\right)^{f\left(\text { Ftr }_{i}^{k}\right)}
$$

where $k$ ranges over all possible features, $f\left(F t r_{i}^{k}\right)$ is a function with value 1 if that feature is present for word $w_{i}$ and with value 0 otherwise, $g\left(\right.$ Synset $\left._{i}, k\right)$ is a parameter for a given synset and feature that is learned from the training corpus with generalized iterative scaling, and $\theta$ is the collection of all these parameters $g\left(\right.$ Synset $\left._{i}, k\right)$. The factor $Z\left(\mathbf{F t r}_{\mathrm{i}}\right)$ is a normalization factor given by $Z\left(\mathbf{F t r}_{\mathrm{i}}\right)=\sum_{i} \prod_{k} g_{k}^{f\left(F t r_{i}^{k}\right)}$. For a given word $w_{i}$ we compute this probability for every possible synset for this word (given by the WordNet dictionary), select the synset with highest probability and assign it to this word.

\subsubsection{Features}

We use a set of features that capture syntactic and semantic properties of the context of the word to be labeled:

Contextual information: The contextual information we use consists of the lemmas of the contexts words on either side of the target word, within a 3 token window and within sentence boundaries.

Syntactic information: We parse a sentence and obtain its grammatical structure using a dependency parser (Nivre et al., 2006) and include grammatical dependencies (e.g. subject, object) and morpho-syntactic features such as part-of-speech, case, number and tense. Features are extracted for all tokens that are related through no more than 3 levels of dependency to the word to be disambiguated.

Semantic information: We use the OpenNLP named entity recognizer ${ }^{9}$ and add the labels of the identified entities (e.g. PERSON, LOCATION, ORGANIZATION) to the feature vector. Additionally we add the label NUMBER every time a number is encountered in the text.

Hypernyms: Hypernyms are retrieved from WordNet and added to the feature set for all noun tokens selected by the contextual and syntactical rules. We include the hypernyms of the most frequent sense, and we include the entire hypernym chain (e.g. MOTOR, MACHINE, DEVICE, INSTRUMENTALITY, ARTIFACT, OBJECT, WHOLE and ENTITY).

In addition to these features traditionally used in WSD systems (e.g. Tratz et al., 2007), we add features derived from the latent words:

Current latent word: The hidden word distribution for the word being disambiguated. We append $|V|$ extra values to the feature vector $\mathbf{f}\left(w_{j}\right)$, containing the probability distribution over the $|V|$ possible values for the hidden variable $h_{i}$.

Latent words in context: The hidden word distributions for all words within a 3 token window of the current word. The vectors with size $|V|$ containing the probability distributions of these words are summed, and the resulting vector is appended to the feature vector.

As a comparison we also consider features derived from classes learned by the full-IBM-predict class-based language model (Goodman, 2001).

\footnotetext{
${ }^{8}$ The parameters of this distribution are the parameters that are found with generalized iterative scaling to maximize the likelihood of the training corpus. We have experimented with $\ell 1$ regularization (e.g. Andrew and Gao, 2007) when determining these parameters, but this did not improve results.

${ }^{9}$ The OpenNLP package is available on http://opennlp.sourceforge.net/.
} 
Table 3

Average accuracy for word sense disambiguation (in $\%, \pm$ standard deviation) using a supervised discriminative classifier with extra features derived from hidden words or classes from a class-based language model.

\begin{tabular}{lllll}
\hline Features & Nouns & Verbs & Adjectives & All \\
\hline Standard & $65.06 \pm 0.52$ & $68.24 \pm 0.60$ & $53.67 \pm 0.55$ & $65.83 \pm 0.48$ \\
thidden words (beam search) & $\mathbf{6 7 . 1 7} \pm 0.61$ & $69.59 \pm 0.51$ & $\mathbf{5 5 . 0 9} \pm 0.45$ & $\mathbf{6 7 . 6 4} \pm 0.40$ \\
thidden words (Gibbs sampling) & $67.13 \pm 0.55$ & $\mathbf{6 9 . 8 7} \pm 0.46$ & $54.75 \pm 0.57$ & $67.33 \pm 0.41$ \\
tclasses & $66.49 \pm 0.43$ & $68.64 \pm 0.46$ & $55.02 \pm 0.45$ & $66.80 \pm 0.43$ \\
\hline
\end{tabular}

Class from current word: The class of the current word.

Classes in context: The classes of all words within a 3 token window of the current word.

Note that the assignment of a class to a particular word is deterministic, i.e. non-probabilistic.

\subsubsection{Evaluation}

We test the word sense disambiguation classifier using 10-fold cross validation on the Semcor corpus (Fellbaum, 1998; Landes et al., 1998), a subset of the English Brown Corpus where all content words are sense-tagged. We divide the corpus in 10 disjunct parts and iteratively train the model on 9 parts and compute the accuracy of our system on the remainder part, ${ }^{10}$ averaging the results. The features for this model are extracted as described above. To estimate the hidden words, we experiment with two LWLM models, one trained with the forward-forward beam search and one trained with Gibbs sampling, both using a 20 million section of the Reuters corpus. Also the full-IBM-predict model was trained on this section. Free parameters of these models are chosen to optimize the perplexity of a held-out corpus. For the full-IBM-predict model the most important parameter, the number of clusters, was found to be optimal at 1250 .

Table 3 gives the accuracy of the discriminative model using the standard features, the standard features augmented with hidden words, and the standard features augmented with classes. We see that both sets of features improve the accuracy of the classifier. The accuracy for the classifier using hidden words features (trained with forward-forward beam search) are $67.17 \%, 69.59 \%$ and $55.09 \%$ for nouns, verbs and adjectives respectively, which are all higher than for the standard supervised classifier. The classifier using features derived from the classes also outperforms the standard classifier, although it performs worse than the classifier that uses hidden words (except for adjectives). Since nouns and verbs make up the majority of the words to be labeled, this classifier also performs lower overall (66.80\%). For nouns and adjectives the difference between the classifier using classes and the classifier using hidden words is not statistically significant, for verbs and overall it is $(p<0.05)$. Furthermore we see that the difference between using the hidden words of the LWLM trained with forward-forward beam search, or trained with Gibbs sampling gives near-identical results. This shows again that these methods perform comparable, although as pointed out earlier, the forward-forward beam search requires far less computing time.

To be able to compare our system with state-of-the-art classifiers, we perform an additional experiment on the test data of the English all words task of the Senseval3 workshop (Snyder and Palmer, 2004). On this dataset our system with hidden words (trained with beam search) achieves $67.61 \%$ overall, which is the best result reported on this dataset to the best of our knowledge, although close to other systems, e.g. Tratz et al. (2007) with 67.0\%.

\subsection{Semantic role labeling}

Semantic roles and semantic frames have a long tradition in linguistics (Fillmore, 1968; Gruber, 1970; Schank and Abelson, 1977). Today, semantic role labels are usually interpreted as annotations of sentence constituents (e.g. noun phrases) that classify the meaning of the constituent with regard to a verb in the sentence (e.g. by assigning a label to parts of the sentence that answers "who", "where", "when", etc. for a particular verb). This provides an analysis of the sentence that can be situated between a grammatical (e.g. syntactic sentence parsing) and semantic analysis (e.g. word sense disambiguation), and offers a semantic structure that generalizes across different syntactic alternations of expressing identical content (Palmer et al., 2005).

$\overline{10}$ To compute the accuracy we use the official scorer of the Senseval3 workshop (Snyder and Palmer, 2004). 


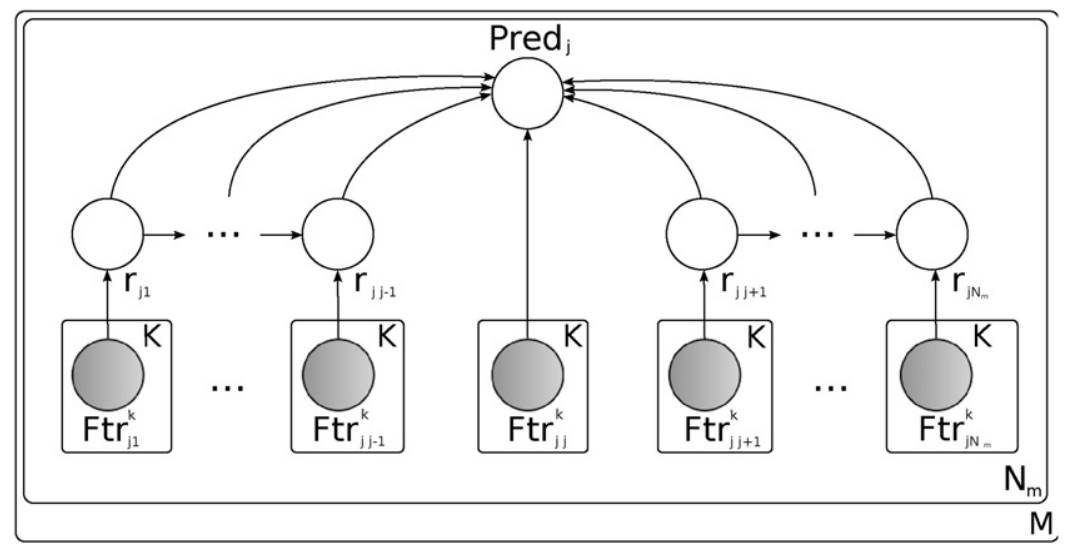

Fig. 4. Discriminative model for SRL. $m$ is the index of the sentence, out of a corpus of $M$ sentences, $j$ is the index of the current verb, out of $N_{m}$ words in the sentence and $k$ is the index of the feature, out of $K$ features.

\subsubsection{PropBank}

A number of definitions for semantic roles have been proposed. We use the PropBank project (Palmer et al., 2005) since it has received the most attention of the NLP community. PropBank defines for a large collection of verbs a set of predicates that reflect the different senses of the verb. The predicates of the verb "run" for example include run.01 "operate, proceed", run.02 "walk quickly" and run.03 "cost". Every predicate label has a number of roles, where label $A 0$ is assigned to the most prominent argument in the sentence ( $A 1$ for unaccusative verbs) and labels $A 1$ to $A 5$ are assigned to other salient arguments for that verb. Although roles are defined for every predicate separately, in reality roles with identical names tend to be syntactically and semantically similar for all predicates, a fact that is exploited to train accurate role classifiers. A small number of arguments is shared among all senses of all verbs, such as temporals $(A M-T M P)$, locatives $(A M-L O C)$ and directionals $(A M-D I R)$. Additional to the role definitions, PropBank has annotated a large training corpus containing approximately 113,000 annotated verbs.

\subsubsection{Discriminative model}

We consider a discriminative model for semantic role labeling (SRL) since discriminative models have been found to outperform generative models for many information extraction tasks, including SRL (Lim et al., 2004). For a given verb on position $j$ and role word on position $i$, we create the feature vector $\mathbf{F t r}_{j i}=\mathbf{F t r}_{j i}^{1} \ldots \mathbf{F t r}_{j i}^{K}$ with length $K$ (see the following section). The model (Fig. 4) assumes that the role label $r_{j i}$ for the word $w_{i}$ is conditioned on the features Ftr $_{j i}$ and on the role label $r_{j i-1}$ of the previous word, and that the predicate label Pred $d_{j}$ for word $w_{j}$ is conditioned on the vector $\mathbf{r}_{j}$ of role labels and on the features $\mathbf{F t r}_{j j}$.

The probabilistic process of this model for a sentence of length $K$ is defined as follows: For $j$ from 1 to $K$ do

For $i$ from 1 to $K$ do

Sample a role $r_{j i}$ from the distribution $P\left(r_{j i} \mid \mathbf{F t r}_{j i}, r_{j i-1}, \theta\right)$

Sample a predicate $\operatorname{Pred}_{j}$ from the distribution $P\left(\operatorname{Pred}_{j} \mid \mathbf{r}_{j}, \theta\right)$

Here $\theta$ is the set of parameters of this model. The probability of a predicate $\operatorname{Pred}_{j}$ and vector of roles $\mathbf{r}_{j}$ given feature vectors $\mathbf{F t r}_{j 1}, \mathbf{F t r}_{j 1}, \ldots$ and $\mathbf{F t r}_{j N_{m}}$ is given by

$$
P\left(\operatorname{Pred}_{j}, \mathbf{r}_{j} \mid \mathbf{F t r}_{j 1}, \ldots, \operatorname{Ftr}_{j N_{m}}, \theta\right)=\prod_{i=1}^{j-1} P\left(r_{j i} \mid \mathbf{F t r}_{j i}, r_{j i-1}\right) \times P\left(\operatorname{Pred}_{j} \mid \mathbf{r}_{j}, \mathbf{F t r}_{j j}\right) \times \prod_{i=j+1}^{N_{m}} P\left(r_{j i} \mid \mathbf{F t r}_{j i}, r_{j i-1}\right)
$$

The probability mass functions $P\left(r_{j i} \mid \mathbf{F t r}_{j i}, r_{j i-1}\right)$ and $P\left(\operatorname{Pred}_{j} \mid \mathbf{r}_{j}, \mathbf{F t r}_{j j}\right)$ are both modeled as exponential distributions (see Section 3.1.1). ${ }^{11}$ This model can be seen as an extension of the standard maximum entropy Markov model (Ratnaparkhi, 1996) with an extra dependency on the predicate label.

\footnotetext{
$\overline{11}$ Also here we experimented with $\ell 1$ regularization, which did not improve results.
} 


\subsubsection{Features}

Every word in the training and test corpus is tagged with its part-of-speech by an automatic tagger (Ciaramita and Altun, 2006, using the Penn Treebank tags) and a syntactic dependency tree is constructed for every sentence by an automatic parser (Nivre et al., 2006). These automatic annotations are, together with the word tokens, converted to a number of features used in our semantic role labeling classifiers. These features (except Split path) have been previously discussed, see for example (Gildea and Jurafsky, 2002; Lim et al., 2004; Thompson et al., 2006). The number in brackets in the following list denotes the number of unique features for that type in the CoNLL 2008 corpus.

Word: Unigram word tokens, including punctuation. $(37,079)$

Stem: Word tokens reduced to their stem, e.g. "walks" $\rightarrow$ "walk". $(28,690)$

POS: The part-of-speech tag for every word, e.g. "NNP". (77)

Neighbor POS's: The concatenated part-of-speech tags of the word before and the word just after the current word, e.g. "RBS_JJR". (1787)

Path: This important feature describes the path through the dependency tree from the current word to the position of

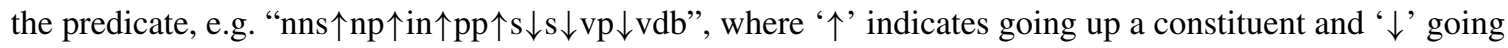
down one constituent. $(829,642)$

Split path: Because of the nature of the path feature, an explosion of unique features is found in a given data set. We reduce this by splitting the path in different parts and using every part as a distinct feature. We split, for

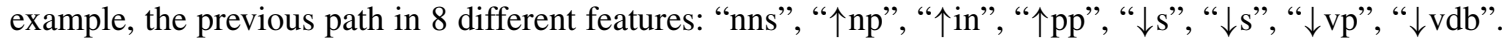
Note that the split path feature includes the POS feature, since the first component of the path is the POS tag for the current word. This feature has not been used previously for semantic role detection. (155)

Child words: The word tokens of the children in the dependency tree of the current word, if any $(34,518)$.

Although most of the described features are independent of the predicate verb, the value of Path and Splitpath differs with regard to the predicate verb. In addition to these standard features we consider features derived from the latent words. For this we estimate the value of the hidden words for both the CoNLL 2008 training and test set. For every word $w_{i}$ to be labeled we then consider the feature:

Current latent word: We append $|V|$ extra values to the feature vector $\mathbf{f}\left(w_{j}\right)$, containing the probability distribution over the $|V|$ possible values for the hidden variable $h_{i}$.

We also test an alternative method where we append the feature vector with the class of the word being labeled:

Class from current word: The class of the current word.

The classes are learned by the full-IBM-predict class-based language model (Goodman, 2001).

\subsubsection{Evaluation}

We perform our experiments on the standard corpus for semantic role labeling used in the CoNLL 2008 shared task (Surdeanu et al., 2008). Although the CoNLL 2008 shared task evaluated semantic role labeling on both verb and noun phrases, we have limited ourselves to the labeling of semantic roles that are arguments of verbs as the latter task is mostly studied in the literature. Most of the sentences in the corpus are from news texts from the Wall Street Journal, while a smaller fraction comes from 15 different sources, including news texts, non-fictional and fictional stories and book reviews. The corpus is split into three disjoint parts, for training (39,279 sentences), testing ${ }^{12}$ (2824 sentences) and a held-out section (1334 sentences). The manual annotations of the sentences are the annotations from the PropBank corpus, but are converted from labels for constituents to labels for head words. For details on this conversion, we refer to Surdeanu et al. (2008).

To evaluate our system we use the evaluation metric used in the CoNLL 2008 shared task, which counts the number of correct labels, i.e. predicate labels and semantic role labels. A predicate label is considered correct if the label

\footnotetext{
12 In the shared task the test set is split in a set with sentences from the Wall Street Journal and a set with sentences from the Brown corpus. In our experiments we report on all sentences from both sets.
} 
Table 4

Results (in average \% F1-measure \pm standard deviation) of 10-fold cross validation for SRL on the CoNLL 2008 training set, comparing the standard supervised classifier with different weakly supervised classifiers, using different portions of the full training set for training. See main text for details.

\begin{tabular}{lllll}
\hline & $5 \%$ & $20 \%$ & $50 \%$ & $100 \%$ \\
\hline Standard & $51.95 \pm 0.62$ & $75.68 \pm 0.31$ & $78.03 \pm 0.41$ & $80.99 \pm 0.37$ \\
thidden words(Gibbs sampling) & $67.62 \pm 0.57$ & $78.20 \pm 0.32$ & $80.65 \pm 0.33$ & $83.53 \pm 0.32$ \\
thidden words(beam search) & $67.15 \pm 0.53$ & $\mathbf{7 8 . 8 4} \pm 0.54$ & $\mathbf{8 0 . 7 6} \pm 0.30$ & $\mathbf{8 3 . 7 2} \pm 0.36$ \\
tclasses & $\mathbf{6 9 . 3 7} \pm 0.48$ & $74.11 \pm 0.35$ & $74.75 \pm 0.48$ & $75.48 \pm 0.38$ \\
\hline
\end{tabular}

corresponds to the label in the manual annotation for that verb. A role label for a particular word is considered correct if the label corresponds to the label in the manual annotation for that word, independently of the label of the verb for that role. For a given test set, $N_{\text {corr }}$ is the number of predicates and role labels that are correctly classified, $N_{\text {man }}$ is the number of predicates and role labels in the manual annotation and $N_{\text {auto }}$ is the number of predicates and role labels in the automatic annotation. Precision, recall and $F 1$-measure are defined as

$$
\text { precision }=\frac{N_{\text {corr }}}{N_{\text {auto }}} ; \quad \text { recall }=\frac{N_{\text {corr }}}{N_{\text {man }}} ; \quad F 1=2 * \frac{\text { precision } * \text { recall }}{\text { precision }+ \text { recall }}
$$

We again train the full-IBM-predict model and LWLM on the 20 million word Reuters corpus. For the LWLM we consider Gibbs sampling or the forward-forward beam search during training. We then find the expected value for the hidden word for all observed words in the CoNLL 2008 corpus. Table 4 shows the results of the different methods on the test set of the CoNLL 2008 shared task. We first experiment with 10-fold cross validation on the CoNLL 2008 training section, where we split the training set in 10 disjunct parts. In 10 iterations we train the classifier on 9 parts and measure performance on the remaining part, averaging the results. To see the influence of the size of the training set, we experiment with different sizes of the training set, using a random selection of $5 \%, 10 \%, 50 \%$ or $100 \%$ of the training set (i.e. percentages from the 9 parts not used for testing).

We see that the method that uses hidden words outperforms the method with standard features across all training sizes. Furthermore, these improvements are larger for smaller training sets, showing that the approach can be applied successfully in a setting where only a small number of training examples is available. When comparing the method that uses hidden words with the method that uses class features, we see that, although the classes result in the best performance for the smallest training sizes, this performance drops for larger training sizes. A possible explanation of this result is the use of the classes employed in this method. By definition the classes merge many words into one cluster, which might lead to good generalization (more important for small training sizes), but can potentially hurt precision (more important for larger training sizes). We see again that the influence of the training method used in the LWLM (forward-forward beam search or Gibbs sampling) has very little influence on the results. The difference in results of the classifiers using the hidden words compared to the standard feature set and the feature set with classes, are all statistically significant $(p<0.05)$. The difference between the results with hidden words trained with beam search and trained with Gibbs sampling are generally not statistically significant.

Although these experiments give us a good idea of the performance of the different feature sets, we perform an additional experiment to compare our best system with state-of-the-art classifiers. We train the classifiers using hidden words (trained with beam search) on 100\% of the training section and test on the CoNLL 2008 test section. This results in an F1-measure of $81.34 \%$. This is a slight improvement of our results reported in Deschacht and Moens (2009), where we used Gibbs sampling with a simplified context model. Compared to state-of-the-art classifiers, we perform worse than the best system (Johansson and Nugues, 2008), which achieved an F1-measure of $84.45 \%$ on this test section. This classifier is however an optimized system that combines an SRL classifier, a set of linguistic restrictions and a global classifier for re-scoring the entire SRL labeling. Our system is much simpler in that it relies on a single classifier and a smaller set of features. We however believe that a more complex system would equally benefit from an extra feature based on the latent words. 


\section{Related work}

In this article we have proposed the LWLM and shown how it (1) improves language modeling and (2) learns latent classes of words that help in information extraction tasks such as word sense disambiguation and semantic role labeling. We will relate these different features to related research in the following sections.

\subsection{Improved language modeling}

Today virtually all language models used in real-life applications (e.g. speech recognition (Bahl et al., 1983) and machine translation (Koehn et al., 2007)) are n-gram models smoothed with a back-off method. Chen and Goodman (1996) performed an extensive evaluation of different smoothing methods and found that (absolute discounted) Kneser-Ney smoothing outperforms all others. In almost three decades not a single smoothing method is proposed with better performance. Some research has focused on developing smoothing methods that are simpler to compute for very large language models (e.g. Brants et al., 2007; Moore and Quirk, 2009). We have shown that our variant of Kneser-Ney smoothing, relative discounted KN, significantly outperforms the widely used absolute discounted variant. Furthermore our method is equally fast, does not employ a different discounting method for lower-order n-grams, does not use the number of unique contexts of a certain n-gram, and can be employed with soft counts.

Some methods outperform Kneser-Ney smoothing by incorporating words further away in the document, such as cache-based language models (Kuhn and De Mori, 1990), methods for automatic model adaptation (Tam and Schultz, 2006) and sentence mixture models (Iyer and Ostendorf, 1997). These models however all use a traditional n-gram language model as a sub-component, which could be replaced by the LWLM, resulting most likely in further improvements.

All other language models that outperform Kneser-Ney and that are limited to the $n-1$ words in the context, map the words to a latent structure. Cluster models assign every word to one cluster and use sequences of clusters to reduce the sampling error (e.g. Brown et al., 1992; Ney et al., 1994). As our method, these models are trained on unannotated texts. Goodman (2001) compared many of these models and showed that the full-IBM-predict clustering model outperforms all others. We have shown in this article that the LWLM model outperforms full-IBM-predict, which we contribute to the fact that cluster models make a hard-assignment of words to a single cluster, while the LWLM allows words to belong to many latent classes.

Another class of latent language models are grammar-based models that use the grammatical structure of a sentence to predict the words that occur in that sentence (Charniak, 2001; Roark, 2001; Uytsel and Compernolle, 2005). As our model, these models face a large computational complexity because of the exponential number of parse trees that need to be considered, and also use a beam-search that disregards the least likely parses. However, a major disadvantage of these models is the need of manually annotated sentences for training, making them unsuitable for many languages and domains.

Another class of language models uses neural networks (Bengio et al., 2003; Schwenk and Gauvain, 2005). These networks map words to continuous feature vectors of lower dimensionality and achieve an improvement over Kneser-Ney smoothing. Also here the computational complexity is very high (e.g. requiring more then 20,000 hrs in Bengio et al. (2003)). In the future we would like to compare these models with the LWLM.

\subsection{Learning latent classes of words}

The LWLM automatically learns classes of related words from their occurrences in a large corpus. It has been long known that words that occur in similar contexts tend to have a similar meaning (Harris, 1954). A large body of work has focused on methods to model the context of a particular word, and to compute a similarity measure on these contexts. Usually a window of words or the direct dependents are taken as features and compared with a cosine measure, Jaccard index or Kullback-Leibler distance (Lin, 1998; Grefenstette, 1994; Grishman and Sterling, 1994; Hearst, 1992). Some authors perform an algebraic dimensionality technique such as singular value decomposition (Schütze, 1992) to further improve the learned distances.

Generative models with latent variables that represent classes of words have been used by NLP researchers for some time. Well-known are unsupervised hidden Markov models that learn part-of-speech tags (Merialdo, 1994). Typically a dictionary provides constraints in the form of possible part-of-speech tags for a large collection of words, which are 
used during the forward-backward algorithm to learn part-of-speech tags for all words in the corpus. Without these constraints, it is hard to learn accurate part-of-speech taggers (Smith and Eisner, 2005), although the importance of correct smoothing methods has also been recognized (Wang and Schuurmans, 2005). Recently, there has also been an interest on learning HMM's with Bayesian techniques such as Gibbs sampling (Goldwater and Griffiths, 2007; Johnson, 2007). A large difference between these methods and the work proposed here is the number of classes: typically previous work aimed to learn part-of-speech tags or equivalent syntactic classes, and thus focuses only on a small ( 20) number of classes. These classes represent syntactic groups of words (e.g. verbs) which are not fine-grained and do not reflect semantic differences. The number of classes in our work is much higher $(\sim 30,000)$ and represent both syntactic and semantic similarities.

The quality of all these different collections of latent classes is hard to compare. One method is to compare the learned classes with an existing dictionary or thesaurus (e.g. Lin, 1998). Most dictionaries however contain only a limited number of words and word senses. A more useful evaluation in our opinion is the usage of the learned classes in real-life NLP tasks, such as performed in Tang et al. (2001) for constituent based parsing, in Koo et al. (2008) for dependency parsing and by Zhao et al. (2009) for multilingual dependency parsing. All these models confirm that classes learned by generative models help in information extraction tasks. We have shown that this is also the case for semantic role labeling and word sense disambiguation with the classes learned by the LWLM. We have furthermore shown that these classes significantly outperform the clusters learned by the full-IBM-predict method.

Finally we would also like to mention the interesting work performed by Collobert and Weston (2008) who propose a convolutional neural network architecture that is jointly trained for language modeling and for a number of different information extraction tasks. During the joint training, a look-up table is learned that maps words to a number of hidden classes. After training, these classes represent syntactically and semantically related words. The authors found that a classifier for semantic role labeling achieved best results when trained jointly as a language model. ${ }^{13}$ Ando and Zhang (2005) have performed experiments with a linear predictor model that incorporates a mapping of the features to a low-dimensional vector, shared across different tasks. They also found that the best results were achieved on a text chunking task when training the model jointly as a language model. These results are also confirmed by our work.

\section{Conclusions and future work}

In this article we have introduced the latent words language model to learn fine-grained, semantic similarities in an unsupervised fashion. This model uses a hidden variable at every position to represent the words that could occur at that position, given the context and given the observed word. We developed two methods to compute the expected value of these variables: the forward-forward beam search and a method based on Gibbs sampling, and we have developed a novel method for training that optimizes the perplexity of this model on unseen texts. We have shown that the interpolated LWLM outperforms other state-of-the-art n-gram models on three different corpora. We attribute this to the fact that the word similarities lessen the sparseness problem of traditional n-gram models. Finally an informal inspection showed that the learned word similarities correlate with human assessment of similar words, and that the hidden words can be used to disambiguate a certain observed word in a certain context.

We have then tested the use of the hidden words as an extra representation of natural language in two information extraction tasks: word sense disambiguation and semantic role labeling and have shown that for both tasks these extra features result in significant improvements over the standard feature set. This improvement was largest for small training sets, showing that this method does successfully reduce the dependency of the supervised model on large training corpora. Because of its simplicity and independence of the specific information extraction task, we expect that this method can be employed almost effortless in other NLP tasks, such as named entity recognition or part-of-speech labeling.

In the future we would like to extend this model in three directions. (1) Develop new techniques to use the hidden words in information extraction methods: Although our experiments show that using the hidden words as probabilistic features results in consistent improvements, we feel that their full potential is not yet reached, especially compared to the improvements of the language model using latent words. An example of an interesting research direction is the

\footnotetext{
${ }^{13}$ We are not able to compare the results of our system with (Collobert and Weston, 2008), since it was tested on a different dataset (Section 23 of the Penn treebank) and uses a different error measure (word error rate) that cannot straightforwardly be translated to a F1-measure.
} 
adaptation of the latent words to a specific task, i.e. find latent words that improve both the likelihood of the language model and the likelihood of the given information extraction task. (2) Apply the LWLM to other data or domains: In many domains ambiguous symbols are encountered, and the context can help to disambiguate these symbols, or to learn a similarity measure between these symbols. Examples are SURF features in the visual domain (Bay et al., 2006) or spectral features in the audio domain (Jurafsky and Martin, 2008). (3) Learn similarities between objects: In many cases the data can be segmented in different objects that group several symbols, such as phrases in texts or objects in images. Often a similarity function between these objects would be more informative than a similarity function between the individual symbols (resp. words in texts or SURF features in images). This similarity measure would have to combine information from the context (i.e. "similar objects occur in similar contexts") and from the symbols describing that object (i.e. "similar objects are composed of similar symbols").

\section{Dissemination}

The implementation of the latent words language model, together with the trained models can be found on http://www.cs.kuleuven.be/groups/liir/software.php. At this page one can also browse the list of automatically learned synonyms and related words.

\section{Acknowledgments}

The presented research was supported by the EU-FP6-IST project CLASS (Cognitive-Level Annotation using Latent Statistical Structure, IST-027978) and by the IWT-SBO project AMASS++(Advanced Multimedia Alignment and Structured Summarization, IWT 060051).

\section{References}

Ando, R., Zhang, T., 2005. A framework for learning predictive structures from multiple tasks and unlabeled data. The Journal of Machine Learning Research 6, 1817-1853.

Andrew, G., Gao, J.,2007. Scalable training of L1-regularized log-linear models. In: Proceedings of the 24th International Conference on Machine Learning. ACM, pp. 33-40.

Bahl, L.R., Jelinek, F., Mercer, R.L., 1983. A maximum likelihood approach to continuous speech recognition. IEEE Transactions on Pattern Analysis and Machine Intelligence 5 (2), 179-190.

Baker, J., 1975. The DRAGON system - an overview. IEEE Transactions on Acoustics, Speech and Signal Processing 23 (1), $24-29$.

Baum, L., Petrie, T., Soules, G., Weiss, N., 1970. A maximization technique occurring in the statistical analysis of probabilistic functions of Markov chains. The Annals of Mathematical Statistics 41 (1), 164-171.

Bay, H., Tuytelaars, T., Van Gool, L.,2006. Surf: speeded up robust features. In: Proceedings of the European Conference on Compute Vision. Springer, pp. 404-417.

Bengio, Y., Ducharme, R., Vincent, P., Janvin, C., 2003. A neural probabilistic language model. Journal of Machine Learning Research 3, $1137-1155$.

Berger, A., Pietra, V., Pietra, S., 1996. A maximum entropy approach to natural language processing. Computational Linguistics 22 (1), 71.

Bishop, C.M., 2006. Pattern Recognition and Machine Learning. Springer New York.

Blei, D.M., Ng, A.Y., Jordan, M.I., 2003. Latent Dirichlet allocation. Journal of Machine Learning Research 3, $993-1022$.

Brants, T., Popat, A.C., Xu, P., Och, F.J., Dean, J.,2007. Large language models in machine translation. In: Proceedings of the 2007 Joint Conference on Empirical Methods in Natural Language Processing and Computational Natural Language Learning (EMNLP-CoNLL). ACL, Prague, Czech Republic, pp. 858-867.

Brants, T., 2000. TnT - a statistical part-of-speech tagger. In: Proceedings of the Sixth Conference on Applied Natural Language Processing, pp. 224-231.

Brown, P., Cocke, J., Pietra, S., Pietra, V., Jelinek, F., Lafferty, J., Mercer, R., Roossin, P., 1990. A statistical approach to machine translation. Computational Linguistics 16 (2), 85.

Brown, P., Mercer, R., Della Pietra, V., Lai, J., 1992. Class-based n-gram models of natural language. Computational Linguistics 18 (4), $467-479$.

Charniak, E.,2001. Immediate-head parsing for language models. In: Proceedings of the 39th Annual Meeting on Association for Computational Linguistics. ACL, pp. 124-131.

Chen, S., Goodman, J.,1996. An empirical study of smoothing techniques for language modeling. In: Proceedings of the 34th Annual Meeting on Association for Computational Linguistics. ACL, pp. 310-318.

Ciaramita, M., Altun, Y.,2006. Broad-coverage sense disambiguation and information extraction with a supersense sequence tagger. In: Proceedings of the 2006 Conference on Empirical Methods in Natural Language Processing. ACL, pp. 594-602.

Collobert, R., Weston, J.,2008. A unified architecture for natural language processing: deep neural networks with multitask learning. In: Proceedings of the 25th International Conference on Machine Learning. ACM, pp. 160-167.

Cortes, C., Vapnik, V., 1995. Support-vector networks. Machine Learning 20 (3), 273-297. 
Deschacht, K., Moens, M.-F.,2009. Semi-supervised semantic role labeling using the Latent Words Language Model. In: Proceedings of the 2009 Conference on Empirical Methods in Natural Language Processing. ACL, Singapore, pp. 21-29.

Fellbaum, C., 1998. WordNet: An Electronic Lexical Database. The MIT Press.

Fillmore, C.J., 1968. The case for case. In: Bach, E., Harms, R. (Eds.), Universals in Linguistic Theory. Rinehart \& Winston.

Gildea, D., Jurafsky, D., 2002. Automatic labeling of semantic roles. Computational Linguistics 28 (3), 245-288.

Goldwater, S., Griffiths, T., 2007. A fully Bayesian approach to unsupervised part-of-speech tagging. Proceedings of the Annual Meeting of the Association for Computational Linguistics 45, 744-751.

Goodman, J.T., 2001. A bit of progress in language modeling, extended version. Technical report, Microsoft Research.

Grefenstette, G., 1994. Explorations in Automatic Thesaurus Discovery. Springer.

Griffiths, T., Steyvers, M., Blei, D., Tenenbaum, J., 2005. Integrating topics and syntax. Advances in Neural Information Processing Systems 17, 537-544.

Grishman, R., Sterling, J., 1994. Generalizing automatically generated selectional patterns. In: Proceedings of the 15th International Conference on Computational Linguistics, pp. 742-747.

Gruber, J., 1970. Studies in Lexical Relations. Indiana University Linguistics Club.

Harris, Z.S., 1954. Distributional structure. Word 10 (23), 146-162.

Hearst, M.,1992. Automatic acquisition of hyponyms from large text corpora. In: Proceedings of the 14th Conference on Computational Linguistics. ACL, pp. 539-545.

Iyer, R., Ostendorf, M.,1997. Transforming out-of-domain estimates to improve in-domain language models. In: Proceedings of the Fifth European Conference on Speech Communication and Technology. ISCA.

Jelinek, F., Bahl, L., Mercer, R., 1975. Design of a linguistic statistical decoder for the recognition of continuous speech. IEEE Transactions on Information Theory 21 (3), 250-256.

Johansson, R., Nugues, P., 2008. Dependency-based syntactic-semantic analysis with PropBank and NomBank. In: Proceedings of the Shared Task Session of CoNLL-2008.

Johnson, M., 2007. Why doesn't EM find good HMM POS-taggers. In: Proceedings of the 2007 Joint Conference on Empirical Methods in Natural Language Processing and Computational Natural Language Learning (EMNLP-CoNLL), pp. 296-305.

Jurafsky, D., Martin, J.H., 2008. Speech and Language Processing. Prentice Hall.

Kemighan, M., Church, K., Gale, W., 1990. A spelling correction program based on a noisy channel model. In: Proceedings of the Thirteenth International Conference on Computational Linguistics, pp. 205-210.

Kilgarriff, A.,1998. Senseval: an exercise in evaluating word sense disambiguation programs. In: Proceedings of LREC. Citeseer, pp. 581-588.

Koehn, P., Hoang, H., Birch, A., Callison-Burch, C., Federico, M., Bertoldi, N., Cowan, B., Shen, W., Moran, C., Zens, R., et al.,2007. Moses: open source toolkit for statistical machine translation. In: Proceedings of the 45th Annual Meeting of the ACL on Interactive Poster and Demonstration Sessions. ACL, pp. 177-180.

Koo, T., Carreras, X., Collins, M.,2008. Simple semi-supervised dependency parsing. In: Proceedings of the Annual Meeting of the Association for Computational Linguistics (ACL). ACL, pp. 595-603.

Krishnamurthy, V., Moore, J., 1993. On-line estimation of hidden Markov model parameters based on the Kullback-Leibler information measure. IEEE Transactions on Signal Processing 41 (8), 2557-2573.

Kudo, T., Matsumoto, Y., 2001. Chunking with support vector machines. In: Proceedings of the Second Meeting of the North American Chapter of the Association for Computational Linguistics on Language Technologies, pp. 1-8.

Kuhn, R., De Mori, R., 1990. A cache-based natural language model for speech reproduction. IEEE Transactions on Pattern Analysis and Machine Intelligence 12 (6), 570-583.

Landes, S., Leacock, C., Tengi, R.I., 1998. Building semantic concordances. In: Fellbaum, C. (Ed.), WordNet: An Electronic Lexical Database. The MIT Press.

Li, W., McCallum, A.,2005. Semi-supervised sequence modeling with syntactic topic models. In: Proceedings of the National Conference on Artificial Intelligence, vol. 20. MIT Press, p. 813.

Lim, J.-H., Hwang, Y.-S., Park, S.-Y., Rim, H.-C.,2004. Semantic role labeling using maximum entropy model. In: Proceedings of the Eighth Conference on Computational Natural Language Learning. ACL, pp. 122-125.

Lin, D., 1998. An information-theoretic definition of similarity. In: Proceedings 15th International Conference on Machine Learning.

MacKay, D.J.C., Peto, L.C.B., 1995. A hierarchical Dirichlet language model. Natural Language Engineering 1, $289-307$.

Marcus, M., Santorini, B., Marcinkiewicz, M., 1994. Building a large annotated corpus of English: the Penn Treebank. Computational Linguistics 19 (2), 313-330

Merialdo, B., 1994. Tagging English text with a probabilistic model. Computational Linguistics 20 (2), 155-171.

Miller, S., Guinness, J., Zamanian, A.,2004. Name tagging with word clusters and discriminative training. In: Proceedings of the 2004 Human Language Technology Conference/North American Chapter of the Association for Computational Linguistics Annual Meeting. ACL.

Mitchell, C., Harper, M., Jamieson, L., et al., 1995. On the complexity of explicit duration HMM's. IEEE Transactions on Speech and Audio Processing 3 (3), 213-217.

Moore, R.C., Quirk, C.,2009. Improved smoothing for n-gram language models based on ordinary counts. In: Proceedings of the ACL-IJCNLP 2009 Conference Short Papers. ACL, Stroudsburg, PA, USA, pp. 349-352.

Murveit, H., Monaco, P., Digalakis, V., Butzberger, J., 1994. Techniques to achieve an accurate real-time large-vocabulary speech recognition system. In: Proceedings of the Workshop on Human Language Technology. ACL, pp. 393-398, ISBN 1558603573.

Ney, H., Essen, U., Kneser, R., 1994. On structuring probabilistic dependences in stochastic language modelling. Computer Speech and Language $8(1), 1-38$. 
Nivre, J., Hall, J., Nilsson, J., 2006. MaltParser: a datadriven parser-generator for dependency parsing. In: Proceedings of the Fifth International Conference on Language Resources and Evaluation, pp. 2216-2219.

Palmer, M., Gildea, D., Kingsbury, P., 2005. The proposition bank: an annotated corpus of semantic roles. Computational Linguistics 31 (1), 71-106.

Ratnaparkhi, A.,1996. A maximum entropy model for part-of-speech tagging. In: Proceedings of the Conference on Empirical Methods in Natural Language Processing. ACL, pp. 133-142.

Roark, B., 2001. Probabilistic top-down parsing and language modeling. Computational Linguistics 27 (2), 249-276, ISSN 0891-2017.

Sang, E., De Meulder, F.,2003. Introduction to the CoNLL-2003 shared task: language-independent named entity recognition. In: Proceedings of CONLL-2003. ACL.

Schütze, H.,1992. Dimensions of meaning. In: Proceedings of the 1992 ACM/IEEE Conference on Supercomputing. IEEE Computer Society Press, pp. 787-796.

Schank, R.C., Abelson, R.P., 1977. Scripts, Plans, Goals and Understanding: An Inquiry into Human Knowledge Structures. L. Erlbaum, Hillsdale, NJ.

Schwenk, H., Gauvain, J.,2005. Connectionist language modeling for large vocabulary continuous speech recognition. In: Proceedings of the IEEE International Conference on Acoustics, Speech, and Signal Processing (ICASSP'05). IEEE.

Shue, L., Dey, S., 2002. Complexity reduction in fixed-lag smoothing for hidden Markov models. IEEE Transactions on Signal Processing 50 (5), 1124-1132.

Smith, N., Eisner, J.,2005. Contrastive estimation: training log-linear models on unlabeled data. In: Proceedings of the 43rd Annual Meeting on Association for Computational Linguistics. Association for Computational Linguistics Morristown, NJ, USA, pp. 354-362.

Snyder, B., Palmer, M., 2004. The English all-words task. In: Proceedings of SENSEVAL, pp. 41-43.

Srihari, R., Baltus, C., 1992. Combining statistical and syntactic methods in recognizing handwritten sentences. In: Proceedings of the AAAI Symposium: Probabilistic Approaches to Natural Language, pp. 121-127.

Surdeanu, M., Johansson, R., Meyers, A., Marquez, L., Nivre, J.,2008. The CoNLL-2008 shared task on joint parsing of syntactic and semantic dependencies. In: Proceedings of the 12th Conference on Computational Natural Language Learning (CoNLL-2008). ACL.

Tam, Y., Schultz, T., 2006. Unsupervised language model adaptation using latent semantic marginals. In: Ninth International Conference on Spoken Language Processing, pp. 2206-2209.

Tang, M., Luo, X., Roukos, S.,2001. Active learning for statistical natural language parsing. In: Proceedings of the 40th Annual Meeting on Association for Computational Linguistics. ACL, pp. 120-127.

Teh, Y.,2006. A hierarchical Bayesian language model based on Pitman-Yor processes. In: Proceedings of the 21st International Conference on Computational Linguistics and the 44th Annual Meeting of the Association for Computational Linguistics. ACL, pp. 985-992.

Thompson, C., Levy, R., Manning, C., 2006. A generative model for FrameNet semantic role labeling. In: Proceedings of the 14th European Conference on Machine Learning.

Tratz, S., Sanfilippo, A., Gregory, M., Chappell, A., Posse, C., Whitney, P.,2007. PNNL: a supervised maximum entropy approach to word sense disambiguation. In: Proceedings of the 4th International Workshop on Semantic Evaluations. ACL, pp. 264-267.

Uytsel, D.H.V., Compernolle, D.V., 2005. Language modeling with probabilistic left corner parsing. Computer Speech \& Language 19 (2), $171-204$.

Wang, Q., Schuurmans, D., 2005. Improved estimation for unsupervised part-of-speech tagging. In: Proceedings of the IEEE International Conference on Natural Language Processing and Knowledge Engineering.

Winograd, T., 1972. Understanding Natural Language. Academic Press.

Winston, P., 1976. The psychology of computer vision. Pattern Recognition 8 (3), 193.

Woodland, P., Odell, J., Valtchev, V., Young, S.,1994. Large vocabulary continuous speech recognition using HTK. In: Proceedings of the IEEE International Conference on Acoustics, Speech, and Signal Processing (ICASSP-94), vol. 2. IEEE.

Yu, S., Kobayashi, H., 2003. An efficient forward-backward algorithm for an explicit-duration hidden Markov model. IEEE Signal Processing Letters 10 (1), 11-14.

Zhao, H., Chen, W., Kazama, J., Uchimoto, K., Torisawa, K.,2009. Multilingual dependency learning: exploiting rich features for tagging syntactic and semantic dependencies. In: Proceedings of the Thirteenth Conference on Computational Natural Language Learning: Shared Task. ACL, pp. 61-66. 